\title{
Interaction of forced Orr-Sommerfeld and Squire modes in a low-order representation of turbulent channel flow
}

\author{
Ryan M. McMullen, Kevin Rosenberg, ${ }^{*}$ and Beverley J. McKeon $\odot^{\dagger}$ \\ Graduate Aerospace Laboratories, California Institute of Technology, Pasadena, California 91125, USA
}

(Received 8 January 2020; accepted 1 July 2020; published 18 August 2020)

\begin{abstract}
A resolvent-based reduced-order representation is used to capture time-averaged secondorder statistics in turbulent channel flow. The recently proposed decomposition of the resolvent operator into two distinct families related to the Orr-Sommerfeld and Squire operators [Rosenberg and McKeon, Efficient representation of exact coherent states of the Navier-Stokes equations using resolvent analysis, Fluid Dyn. Res. 51, 011401 (2019)] results in dramatic improvement in the ability to match all components of the energy spectra and the $u v$ cospectrum. The success of the new representation relies on the ability of the Squire modes to compete with the vorticity generated by Orr-Sommerfeld modes, which is demonstrated by decomposing the statistics into contributions from each family. It is then shown that this competition can be used to infer a phase relationship between the two sets of modes. Additionally, the relative Reynolds number scalings for the two families of resolvent weights are derived for the universal classes of resolvent modes presented by Moarref et al. [Moarref, Sharma, Tropp, and McKeon, Model-based scaling of the streamwise energy density in high-Reynolds-number turbulent channels, J. Fluid Mech. 734, 275 (2013)]. These developments can be viewed as a starting point for further modeling efforts to quantify nonlinear interactions in wall-bounded turbulence.
\end{abstract}

DOI: 10.1103/PhysRevFluids.5.084607

\section{INTRODUCTION AND BACKGROUND}

Techniques borrowed from developments in nonmodal stability theory [1-3] have been met with much success in the analysis of wall-bounded turbulent shear flows. For example, analyses of the Navier-Stokes equations (NSE) linearized about the turbulent mean velocity predict the spanwise length scales associated with the near-wall cycle and large-scale structures in the outer region of the flow from both a transient growth [4-6] and energy amplification of harmonic and stochastic forcing perspective [6,7]. More recently, the linearized equations have been used to develop linear estimators [8-10] and compute impulse responses [11] that qualitatively reproduce the coherence and self-similarity of large-scale motions.

As turbulence is an inherently nonlinear phenomenon, a complete model must account for nonlinear interactions. A common approach to incorporate the effects of nonlinearity into linear models is to augment the linearized equations with an eddy viscosity, such that the turbulent mean profile is fixed as an equilibrium solution of the modified mean momentum equation [4-9,11]. While this approach justifies linearization about the turbulent mean profile, it precludes the study of finite-amplitude fluctuations, since their nonlinear interactions would feed back on and further alter the mean. Instead of using an eddy viscosity, Zare et al. [12] considered colored-in-time stochastic forcing of the linearized NSE in the problem of completing partially known second-order statistics.

\footnotetext{
*Present address: US Air Force Research Laboratory, Wright-Patterson Air Force Base, OH 45433, USA.

†mckeon@caltech.edu
} 
Notably, they demonstrated that their approach can be equivalently represented as a low-rank modification of the original equations.

In a different approach to dealing with nonlinearity, the resolvent analysis framework introduced by McKeon and Sharma [13] retains the nonlinear term and interprets it as endogenous forcing of the linear dynamics through triadic interactions with the velocity fluctuations at other wave-numberfrequency combinations. This framework eliminates the need to incorporate an eddy viscosity for self-consistency, as no linearization is performed. Landahl [14] arrived at a similar formulation, deriving a forced Orr-Sommerfeld (OS) equation in the study of wall-pressure fluctuations. However, Landahl sought approximate solutions in eigenfunction expansions, while resolvent analysis uses a gain-based approach, in which the fluctuations are expressed in terms of the most linearly amplified functions, obtained from a singular value decomposition (SVD) of the resolvent operator associated with the linearized NSE.

Closure of the loop requires determination of the forcing such that it yields the correct velocity Fourier modes, as well as the mean velocity profile, which is assumed known. The forcing can be expanded as a sum over a set of basis functions such that the unknowns are the complex amplitudes, called the resolvent weights. An exact equation for the weights can be formulated [15], though it is intractable to solve for most complex flows of interest. Consequently, there have been previous attempts to estimate the weights from data, e.g., by using either a single time series or power spectral density of the velocity fluctuations [16,17]. Alternatively, Moarref et al. [18] used convex optimization to compute the weights for a resolvent-based low-order representation of time-averaged velocity spectra that minimize the deviation from spectra obtained from a direct numerical simulation (DNS) of $\mathrm{Re}_{\tau}=2003$ channel flow [19]. Towne et al. [20] established a link between resolvent analysis and spectral proper orthogonal decomposition (SPOD) and showed that if the resolvent weights are treated as stochastic quantities their covariance matrix can be calculated from the SPOD modes, which inherently rely on statistical data.

In special cases where full information of the nonlinear forcing is available, such as for exact coherent states (ECS), the resolvent weights can be computed exactly by projecting the forcing onto the aforementioned set of basis functions [21]. For ECS families in channel and pipe flow, which come in pairs of upper and lower branch solutions, the lower branch ones are typically well represented by only a few resolvent modes, whereas many of the upper branch solutions are not captured as efficiently. Furthermore, the wall-normal and spanwise velocity components converge much more slowly than the streamwise velocity. However, an alternative decomposition of the resolvent operator recently proposed by Rosenberg and McKeon [22] yields two families of modes related to the Orr-Sommerfeld and Squire (SQ) operators from classical linear stability theory. By projecting the same channel ECS, they demonstrated that the new sets of basis functions enable a much more compact representation of both branches of solutions, and, notably, all three velocity components converge at roughly the same rate. Subsequent analysis attributed the improved efficacy of the alternative decomposition to the isolation of the wall-normal velocity response into the Orr-Sommerfeld modes, such that the Squire wall-normal vorticity is free to interact with that generated by the Orr-Sommerfeld modes [23].

While the utility of the decomposition into Orr-Sommerfeld and Squire modes for highly simplified flows like ECS has been established, an open question is whether or not it remains relevant for high Reynolds number turbulence. In the present paper, it is shown that the second-order statistics of turbulent channel flow can be accurately represented using a low-order approximation based on this framework. It is additionally shown that the vorticities produced by the Orr-Sommerfeld and Squire modes act to oppose each other, and this observation reveals information about how the resolvent weights for the two families scale relative to each other with Reynolds number. Altogether, these insights point to a mechanism in turbulent channel flow that is important for low-order modeling efforts. 


\section{FORMULATION}

\section{A. Resolvent analysis of turbulent channel flow}

The approach is based on the resolvent analysis framework of McKeon and Sharma [13], in which the incompressible NSE,

$$
\begin{aligned}
\partial_{t} \tilde{\boldsymbol{u}}+(\tilde{\boldsymbol{u}} \cdot \nabla) \tilde{\boldsymbol{u}} & =-\nabla \tilde{p}+\mathrm{Re}_{\tau}^{-1} \nabla^{2} \tilde{\boldsymbol{u}}, \\
\nabla \cdot \tilde{\boldsymbol{u}} & =0,
\end{aligned}
$$

here nondimensionalized using the friction velocity $u_{\tau}$ and channel half height $h$, are first Reynolds decomposed as $\tilde{\boldsymbol{u}}=\boldsymbol{U}+\boldsymbol{u}$, where $\boldsymbol{U}=\left(\begin{array}{lll}U(y) & 0 & 0\end{array}\right)^{\mathrm{T}}$ is the turbulent mean velocity profile and $\boldsymbol{u}$ are the fluctuations about the mean, and then Fourier transformed in the homogeneous wall-parallel and temporal directions $x, z$, and $t$, resulting in equations for the Fourier coefficients, denoted by $(\hat{\bullet})$. For each wave-number-frequency triplet $\left(\begin{array}{lll}k_{x} & k_{z} & \omega\end{array}\right)^{\mathrm{T}} \neq \mathbf{0}$, we have

$$
\begin{aligned}
-i \omega \hat{\boldsymbol{u}}+(\boldsymbol{U} \cdot \nabla) \hat{\boldsymbol{u}}+(\hat{\boldsymbol{u}} \cdot \nabla) \boldsymbol{U}+\nabla \hat{p}-\operatorname{Re}_{\tau}^{-1} \nabla^{2} \hat{\boldsymbol{u}} & =\hat{\boldsymbol{f}}, \\
\nabla \cdot \hat{\boldsymbol{u}} & =0,
\end{aligned}
$$

where $\boldsymbol{f}=-(\boldsymbol{u} \cdot \nabla) \boldsymbol{u}+\langle(\boldsymbol{u} \cdot \nabla) \boldsymbol{u}\rangle$, with $\langle\cdot\rangle$ denoting an averaged quantity, is interpreted as a forcing that drives the dynamics linear in $\hat{\boldsymbol{u}}$. The pressure can be projected out of Eq. (2) using the standard mapping to wall-normal velocity $\hat{v}$ and wall-normal vorticity $\hat{\eta}=i k_{z} \hat{u}-i k_{x} \hat{w}$. The equations are then concisely written as

$$
\left(\begin{array}{l}
\hat{v} \\
\hat{\eta}
\end{array}\right)=\mathcal{H}\left(k_{x}, k_{z}, \omega\right) \hat{\mathbf{g}}
$$

where

$$
\mathcal{H}=\left(\begin{array}{cc}
-i \omega-\Delta^{-1} \mathcal{L}^{\mathrm{OS}} & 0 \\
-i k_{z} U^{\prime} & -i \omega-\mathcal{L}^{\mathrm{SQ}}
\end{array}\right)^{-1}
$$

is the resolvent operator, $\Delta=\mathcal{D}^{2}-k^{2}, \mathcal{D}=d / d y, k^{2}=k_{x}^{2}+k_{z}^{2}$, and $U^{\prime}=\mathcal{D} U$. Additionally,

$$
\begin{aligned}
& \mathcal{L}^{\mathrm{OS}}=i k_{x}\left(U^{\prime \prime}-U \Delta\right)+\mathrm{Re}_{\tau}^{-1} \Delta^{2}, \\
& \mathcal{L}^{\mathrm{SQ}}=-i k_{x} U+\mathrm{Re}_{\tau}^{-1} \Delta
\end{aligned}
$$

are the OS and SQ operators, respectively. The forcing term $\hat{\boldsymbol{g}}=\left(\begin{array}{ll}\hat{g}_{v} & \hat{g}_{\eta}\end{array}\right)^{\mathrm{T}}$ in Eq. (3) is related to $\hat{f}$ via

$$
\hat{\boldsymbol{g}}=\underbrace{\left(\begin{array}{ccc}
-i k_{x} \Delta^{-1} \mathcal{D} & -k^{2} \Delta^{-1} & -i k_{z} \Delta^{-1} \mathcal{D} \\
i k_{z} & 0 & -i k_{x}
\end{array}\right)}_{\mathcal{B}} \hat{\boldsymbol{f}},
$$

such that $\hat{g}_{v}=-\Delta^{-1}\left(i k_{x} \mathcal{D} \hat{f_{x}}+k^{2} \hat{f}_{y}+i k_{z} \mathcal{D} \hat{f_{z}}\right)$ and $\hat{g}_{\eta}=i k_{z} \hat{f}_{x}-i k_{x} \hat{f}_{z}$. As pointed out by Rosenberg and McKeon [22], only the solenoidal part of $\hat{\boldsymbol{f}}$ contributes to $\hat{\boldsymbol{g}}$, since the irrotational component lies in the null space of $\mathcal{B}$.

With the aim of obtaining a low-order representation of Eq. (3), we compute the Schmidt decomposition of $\mathcal{H}$ :

$$
\mathcal{H}=\sum_{j=1}^{\infty} \boldsymbol{\psi}_{j} \sigma_{j}\left\langle\cdot, \boldsymbol{\phi}_{j}\right\rangle,
$$

where $\sigma_{j} \geqslant \sigma_{j+1} \geqslant 0 \forall j$ are the singular values, and $\boldsymbol{\psi}_{j}$ and $\boldsymbol{\phi}_{j}$ are the left and right singular vectors, respectively. Since the $\psi_{j}$ are a basis for the output space, i.e., the space to which the response 
$\left(\begin{array}{ll}\hat{v} & \hat{\eta}\end{array}\right)^{\mathrm{T}}$ belongs, they are referred to as response modes; similarly, the $\boldsymbol{\phi}_{j}$ are referred to as the forcing modes. The Schmidt decomposition applies to linear operators on infinite-dimensional vector spaces. For the finite-dimensional matrix approximation obtained from numerically discretizing the operator, this becomes the SVD, which we refer to hereafter for simplicity. As is evident from Eq. (7), the SVD depends on the choice of inner product $\langle\cdot, \cdot\rangle$. On both the input and output spaces we adopt the standard kinetic-energy inner product [24]:

$$
\left\langle\boldsymbol{x}_{1}, \boldsymbol{x}_{2}\right\rangle=\int_{-1}^{1} \boldsymbol{x}_{2}^{*} \mathcal{Q} \boldsymbol{x}_{1} d y
$$

where $(\cdot)^{*}$ denotes the conjugate transpose and

$$
\mathcal{Q}=\frac{1}{k^{2}}\left(\begin{array}{cc}
-\Delta & 0 \\
0 & 1
\end{array}\right)
$$

The left and right singular vectors are orthonormal with respect to this inner product, i.e., $\left\langle\boldsymbol{\psi}_{j}, \boldsymbol{\psi}_{k}\right\rangle=$ $\left\langle\boldsymbol{\phi}_{j}, \boldsymbol{\phi}_{k}\right\rangle=\delta_{j k}$, where $\delta_{j k}$ is the Kronecker delta.

The desired low-order approximation of Eq. (3) is obtained by truncating the sum in Eq. (7):

$$
\left(\begin{array}{l}
\hat{v} \\
\hat{\eta}
\end{array}\right) \approx \sum_{j=1}^{N} \boldsymbol{\psi}_{j} \sigma_{j} \chi_{j},
$$

for some $N \geqslant 1$. We refer to this as the rank- $N$ approximation. The $\chi_{j}=\left\langle\hat{\boldsymbol{g}}, \boldsymbol{\phi}_{j}\right\rangle$ are called the resolvent weights and quantify how much of the forcing $\hat{\boldsymbol{g}}$ is in the direction $\boldsymbol{\phi}_{j}$. For broadband forcing in $y$, i.e., $\chi_{j}=\chi \forall j$, Eq. (10) is optimal in the norm induced by the inner product in Eq. (8). Furthermore, if $\sum_{j=1}^{N} \sigma_{j}^{2} \approx \sum_{j=1}^{\infty} \sigma_{j}^{2}$ for relatively small $N, \mathcal{H}$ is said to be effectively low rank. It has been shown that this property holds for a large portion of spectral space that is energetically significant [25], and this low-rank behavior has previously been exploited to model salient features in wall-bounded turbulence $[13,25,26]$. However, the assumption of broadband forcing is in general not valid. For the case of structured forcing, Eq. (10) is an accurate approximation of the full system, provided the forcing is not too aligned in any of the truncated directions.

Finally, in order to compute the second-order velocity statistics, the velocity $\hat{\boldsymbol{u}}$ is recovered from the response via

$$
\hat{\boldsymbol{u}}=\frac{1}{k^{2}}\left(\begin{array}{cc}
i k_{x} \mathcal{D} & -i k_{z} \\
k^{2} & 0 \\
i k_{z} \mathcal{D} & i k_{x}
\end{array}\right)\left(\begin{array}{l}
\hat{v} \\
\hat{\eta}
\end{array}\right) .
$$

\section{B. Orr-Sommerfeld and Squire decomposition of the resolvent}

As discussed in Sec. II A, the decomposition of $\mathcal{H}$ given in Eq. (7), hereafter referred to as the standard resolvent decomposition, is optimal in the kinetic-energy norm induced by the inner product in Eq. (8). However, in wall-bounded turbulence the kinetic energy is often dominated by the streamwise velocity, which means that all three velocity components may not be approximated uniformly well $[18,21]$. In such situations, an alternative decomposition that more faithfully represents the underlying dynamics may be desirable. This idea has been explored previously by Juttijudata et al. [27], who transformed near-wall data from turbulent channel flow into Squire's coordinate system and then performed POD on modes associated with the streamwise streaks and rolls separately. While the resulting basis functions are energetically suboptimal compared to those from standard POD, they demonstrate that the reconstruction of wall-normal, spanwise, and Reynolds shear stress statistics improves substantially. 
In a similar spirit, Rosenberg and McKeon [22], proposed the following alternative decomposition of $\mathcal{H}$. Note that Eq. (3) can be rewritten as

$$
\left(\begin{array}{l}
\hat{v} \\
\hat{\eta}
\end{array}\right)=\left(\begin{array}{cc}
\mathcal{H}_{v v} & 0 \\
\mathcal{H}_{\eta v} & \mathcal{H}_{\eta \eta}
\end{array}\right)\left(\begin{array}{l}
\hat{g}_{v} \\
\hat{g}_{\eta}
\end{array}\right)
$$

where

$$
\begin{aligned}
& \mathcal{H}_{v v}=\left(-i \omega-\Delta^{-1} \mathcal{L}^{\mathrm{OS}}\right)^{-1}, \\
& \mathcal{H}_{\eta \eta}=\left(-i \omega-\mathcal{L}^{\mathrm{SQ}}\right)^{-1}, \\
& \mathcal{H}_{\eta v}=-i k_{z} \mathcal{H}_{\eta \eta} U^{\prime} \mathcal{H}_{v v} .
\end{aligned}
$$

Apparently, $\mathcal{H}_{v v}$ and $\mathcal{H}_{\eta v}$ are forced by $\hat{g}_{v}$ only, while $\mathcal{H}_{\eta \eta}$ is forced by $\hat{g}_{\eta}$ only. This motivates the separation of the response $\left(\begin{array}{ll}\hat{v} & \hat{\eta}\end{array}\right)^{\mathrm{T}}$ into two distinct families:

$$
\begin{aligned}
\left(\begin{array}{c}
\hat{v} \\
\hat{\eta}^{\mathrm{OS}}
\end{array}\right) & =\left(\begin{array}{c}
\mathcal{H}_{v v} \\
\mathcal{H}_{\eta v}
\end{array}\right) \hat{g}_{v}, \\
\hat{\eta}^{\mathrm{SQ}} & =\mathcal{H}_{\eta \eta} \hat{g}_{\eta} .
\end{aligned}
$$

In the following, we refer to the family of modes in Eq. (14a) as OS modes and the family in Eq. (14b) as SQ modes. The separation of $\hat{\eta}$ into two distinct families is common practice in linear stability analysis, where the SQ and OS modes are, respectively, the homogeneous and particular solutions of the Squire equation: $\left(-i \omega-\mathcal{L}^{\mathrm{SQ}}\right) \hat{\eta}=-i k_{z} U^{\prime} \hat{v}$ [24]. That is, the OS modes can be interpreted as a response to the wall-normal velocity. This interpretation still holds in the nonlinear setting, since the second component of Eq. (14a) can be written as $\hat{\eta}^{\mathrm{OS}}=-i k_{z} \mathcal{H}_{\eta \eta} U^{\prime} \hat{v}$. However, the SQ modes are no longer the homogeneous solutions, but are now interpreted as the response to forcing by $\hat{g}_{\eta}$.

Note that only the OS modes contain a $\hat{v}$ response, such that the SQ modes contribute only to the $\hat{\eta}$ response, i.e., to the wall-parallel velocity components. There is thus the potential for interaction between the OS and SQ vorticity in ways that are not admitted by the standard resolvent decomposition. This fact is of central importance for the OS-SQ resolvent decomposition, and it will be demonstrated in Sec. III A that this drastically improves the accuracy of a low-order resolventbased representation of the second-order statistics for turbulent channel flow.

An SVD of each operator in Eq. (14) is performed separately, and the resulting decomposition is referred to as the OS-SQ decomposition of the resolvent. The approximation of the response becomes

$$
\left(\begin{array}{l}
\hat{v} \\
\hat{\eta}
\end{array}\right) \approx \sum_{j=1}^{N^{\mathrm{OS}}} \boldsymbol{\psi}_{j}^{\mathrm{OS}} \sigma_{j}^{\mathrm{OS}} \chi_{j}^{\mathrm{OS}}+\sum_{k=1}^{N^{\mathrm{SQ}}} \boldsymbol{\psi}_{k}^{\mathrm{SQ}} \sigma_{k}^{\mathrm{SQ}} \chi_{k}^{\mathrm{SQ}}
$$

Note that Eq. (15) is now a sum of $N^{\mathrm{OS}}+N^{\mathrm{SQ}}$ terms. Furthermore, while the left and right singular vectors of each family still comprise orthonormal sets with respect to the inner product given in Eq. (8), it is not guaranteed that modes belonging to different families are orthonormal, e.g., $\left\langle\boldsymbol{\psi}_{j}^{\mathrm{OS}}, \boldsymbol{\psi}_{k}^{\mathrm{SQ}}\right\rangle \neq \delta_{j k}$ in general.

\section{Empirical determination of the resolvent weights via convex optimization}

The singular values and vectors are computed directly from the resolvent operator, which depends only on the (assumed known) mean velocity profile $U$, whereas computation of the weights requires solution of a nonlinear programming problem [15]. This can be done exactly in special cases, such as for ECSs [23]. However, it rapidly becomes intractable with an increasing number of degrees of freedom, and, to our knowledge, fully turbulent flows remain out of reach. 
Consequently, several attempts have been made to determine the weights empirically [12,1618,20]. In particular, Moarref et al. [18], used convex optimization to compute the weights that minimize the deviation between a resolvent-based representation of the energy spectra and DNS data. We take the same approach here and largely adopt their formulation, with the major exception that we employ the OS-SQ decomposition discussed in Sec. II B. That is, for given $N^{\mathrm{OS}}$ and $N^{\mathrm{SQ}}$, we attempt to approximate the DNS statistics using the approximation given in Eq. (15).

As introduced by Moarref et al. [18], the resolvent three-dimensional streamwise energy spectra are

$$
E_{r}\left(y, k_{x}, k_{z}, c\right)=\operatorname{Re}\left\{\operatorname{tr}\left(\boldsymbol{A}_{r} \boldsymbol{X}\right)\right\},
$$

with $r \in\{u u, v v, w w, u v\}$, and where $\operatorname{Re}\{\cdot\}$ is the real part of a complex number $\operatorname{and} \operatorname{tr}(\cdot)$ is the matrix trace. Note that we have chosen to parametrize the spectra in terms of the wave speed $c=\omega / k_{x}$ since resolvent modes tend to be localized about the critical layers $y_{c}$, where $U\left(y_{c}\right)=c$ [13], and it has been observed experimentally that the range of energetic wave speeds is relatively compact, with the most energetic motions typically being confined to the range $8 \lesssim c \lesssim U_{\mathrm{CL}}$ [28], where $U_{\mathrm{CL}}$ is the mean centerline velocity. In Eq. (16), the matrix $\boldsymbol{A}_{u u}$, for example, with entries

$$
\boldsymbol{A}_{u u, i j}=\sigma_{i} \sigma_{j} \hat{u}_{i} \hat{u}_{j}^{*},
$$

represents the contributions of the singular values and response modes and can be determined $a$ priori from the SVD of the resolvent. The matrix $\boldsymbol{X}$, with entries

$$
\boldsymbol{X}_{i j}=\chi_{i}^{*} \chi_{j}
$$

is the weights matrix. Apparent from this definition is that $\boldsymbol{X}^{\mathrm{T}}=\chi \chi^{*} \succeq \mathbf{0}$, where $\chi$ is the vector of weights and $\succeq$ denotes the Löwner order, i.e., $\boldsymbol{X}$ is a rank-1 positive-semidefinite matrix. The OS-SQ decomposition is incorporated into this framework simply by partitioning the $\boldsymbol{A}_{r}$ and $\boldsymbol{X}$ matrices as

$$
\boldsymbol{A}_{r}=\left(\begin{array}{ll}
\boldsymbol{A}_{r}^{\mathrm{OS} / \mathrm{OS}} & \boldsymbol{A}_{r}^{\mathrm{OS} / \mathrm{SQ}} \\
\boldsymbol{A}_{r}^{\mathrm{SQ} / \mathrm{OS}} & \boldsymbol{A}_{r}^{\mathrm{SQ} / \mathrm{SQ}}
\end{array}\right), \quad \boldsymbol{X}=\left(\begin{array}{cc}
\boldsymbol{X}^{\mathrm{OS} / \mathrm{OS}} & \boldsymbol{X}^{\mathrm{OS} / \mathrm{SQ}} \\
\boldsymbol{X}^{\mathrm{OS} / \mathrm{SQ} *} & \boldsymbol{X}^{\mathrm{SQ} / \mathrm{SQ}}
\end{array}\right),
$$

where the superscript $\mathrm{X} / \mathrm{Y}$ denotes the family of the $i$ th and $j$ th mode, respectively, in Eqs. (17) and (18).

The goal is to compute the weights matrix such that the deviation between the wave-speedintegrated resolvent spectra in Eq. (16) and time-averaged DNS spectra is minimized. After discretization of the wave-speed range $c \in\left[0, U_{\mathrm{CL}}\right]$, this can be formally cast as the following optimization problem: For fixed $k_{x}$ and $k_{z}$,

$$
\begin{aligned}
& \underset{\left\{\boldsymbol{X}_{l}\right\}_{l=1,2, \ldots, N_{c}}, e}{\operatorname{minimize}} e \\
& \text { subject to } \frac{\left\|E_{r}^{\mathrm{DNS}}-\sum_{l=1}^{N_{c}} k_{x} d c \operatorname{Re}\left\{\operatorname{tr}\left(\boldsymbol{A}_{r, l} \boldsymbol{X}_{l}\right)\right\}\right\|^{2}}{\left\|E_{r}^{\mathrm{DNS}}\right\|^{2}} \leqslant e \\
& \quad \boldsymbol{X}_{l} \succeq \mathbf{0}, l=1,2, \ldots, N_{c},
\end{aligned}
$$

where the subscript $l$ denotes a quantity evaluated at $c=c_{l}$. Note that the norm $\|\cdot\|$ is not the one induced by Eq. (8). It is defined as

$$
\|f\|^{2}=\int_{y_{\min }^{+}}^{y_{\max }^{+}}\left|f\left(\log y^{+}\right)\right|^{2} d \log y^{+}
$$

and is designed to penalize deviations across the channel equally [18]. Thus deviations from the DNS spectra are penalized for $5 \leqslant y_{\text {min }}^{+} \leqslant y^{+} \leqslant y_{\text {max }}^{+}<\operatorname{Re}_{\tau}$.

Equation (20) is a semidefinite program for the weights matrices $\boldsymbol{X}_{l}$ and can therefore be solved efficiently using a convex optimization software package. Note that imposing the rank-1 constraint 
TABLE I. Resolutions of the DNS from which spectra were obtained for the optimization.

\begin{tabular}{lrrr}
\hline \hline $\operatorname{Re}_{\tau}$ & $N_{x}$ & $N_{y}$ & $N_{z}$ \\
\hline 185 (Channelflow, present paper) & 384 & 129 & 128 \\
$934[19]$ & 1024 & 385 & 768 \\
$2003[19]$ & 2048 & 635 & 1536 \\
$4219[32]$ & 1024 & 1081 & 1024 \\
\hline \hline
\end{tabular}

on the $\boldsymbol{X}_{l}$ would make Eq. (20) nonconvex. Moarref et al. [18] employed an iterative rank-reduction procedure to recover rank-1 matrices from the full-rank solution [29]. However, we do not employ this algorithm here and instead choose to work with the full-rank weights matrices. In this case, the $\boldsymbol{X}_{l}$ can be interpreted as the covariance matrices of the weights, similar to Towne et al. [20]. Finally, since the optimization is performed for second-order statistics, the present approach does not provide phase information about modes with different wave numbers. This means that the computed weights do not yield a closed, self-consistent system, as such information is necessary to recover the mean velocity profile as well as the fluctuations. Extension of the method to incorporate phase is a direction for future work.

\section{Numerical details}

The resolvent operators are discretized in MATLAB using a Chebyshev pseudospectral method [30]; all results presented here use 203 Chebyshev polynomials. The SVDs of the discretized operators are performed with a random matrix algorithm, which is faster than MATLAB's built-in svd() [31]. The time-averaged two-dimensional (2D) DNS spectra for $\operatorname{Re}_{\tau}=934$ and 2003 are obtained from Hoyas and Jiménez [19], and those for $\operatorname{Re}_{\tau}=4219$ are obtained from Lozano-Durán and Jiménez [32]. Additionally, spectra were generated for $\operatorname{Re}_{\tau}=185$ using CHANNELFLOW [33]. The resolutions of all the DNS considered are given in Table I.

For the results presented in Sec. III A, which focus on $\mathrm{Re}_{\tau}=2003$, the DNS spectra are interpolated onto a grid of $N_{k_{x}}=30$ by $N_{k_{z}}=31$ logarithmically spaced wave numbers, which is sufficient to reproduce the general shape of the spectra. Furthermore, it has recently been shown that statistics such as the $u v$ Reynolds stress can be accurately reproduced even when retaining only about $2 \%$ of the wave numbers from DNS [34]. Both the spectra and resolvent modes are interpolated onto a common grid of $N_{y}=60$ logarithmically spaced points in the wall-normal direction, and the wave-speed range $c \in\left[0, U_{\mathrm{CL}}\right]$ is discretized into $N_{c}=100$ linearly spaced wave speeds. The optimization problem Eq. (20) is then solved with CVX [35]. The results are insensitive to further increases in $N_{y}$ and $N_{c}$ [18].

\section{ANALYSIS OF THE OPTIMIZED SPECTRA}

\section{A. Reconstruction of time-averaged statistics}

The accuracy of the optimized spectra is evaluated by comparing them to the time-averaged statistics from the DNS for $\operatorname{Re}_{\tau}=2003$. The premultiplied one-dimensional (1D) $k_{x}$ spectra,

$$
k_{x} E_{r}\left(y, k_{x}\right)=\int_{k_{z, \min }}^{k_{z, \max }} \int_{0}^{U_{\mathrm{CL}}} k_{x}^{2} E_{r}\left(y, k_{x}, k_{z}, c\right) d c d k_{z},
$$

using $N^{\mathrm{OS}}=N^{\mathrm{SQ}}=3$ modes, i.e., six modes per wave-number-frequency triplet, are compared to the DNS in the right column of Fig. 1, which is plotted in terms of $\lambda_{x}^{+}=2 \pi / k_{x}^{+}$. Clearly, $N^{\mathrm{OS}}=$ $N^{\mathrm{SQ}}=3$ modes is sufficient to accurately reproduce the spectra since the overall agreement between the resolvent and DNS spectra is very good, and, in particular, the peaks are captured almost exactly. The only significant discrepancies are in $k_{x} E_{u u}$ at large $\lambda_{x}^{+}$and $y^{+} \lesssim 100$ and $-k_{x} E_{u v}$ at large $\lambda_{x}^{+}$ 

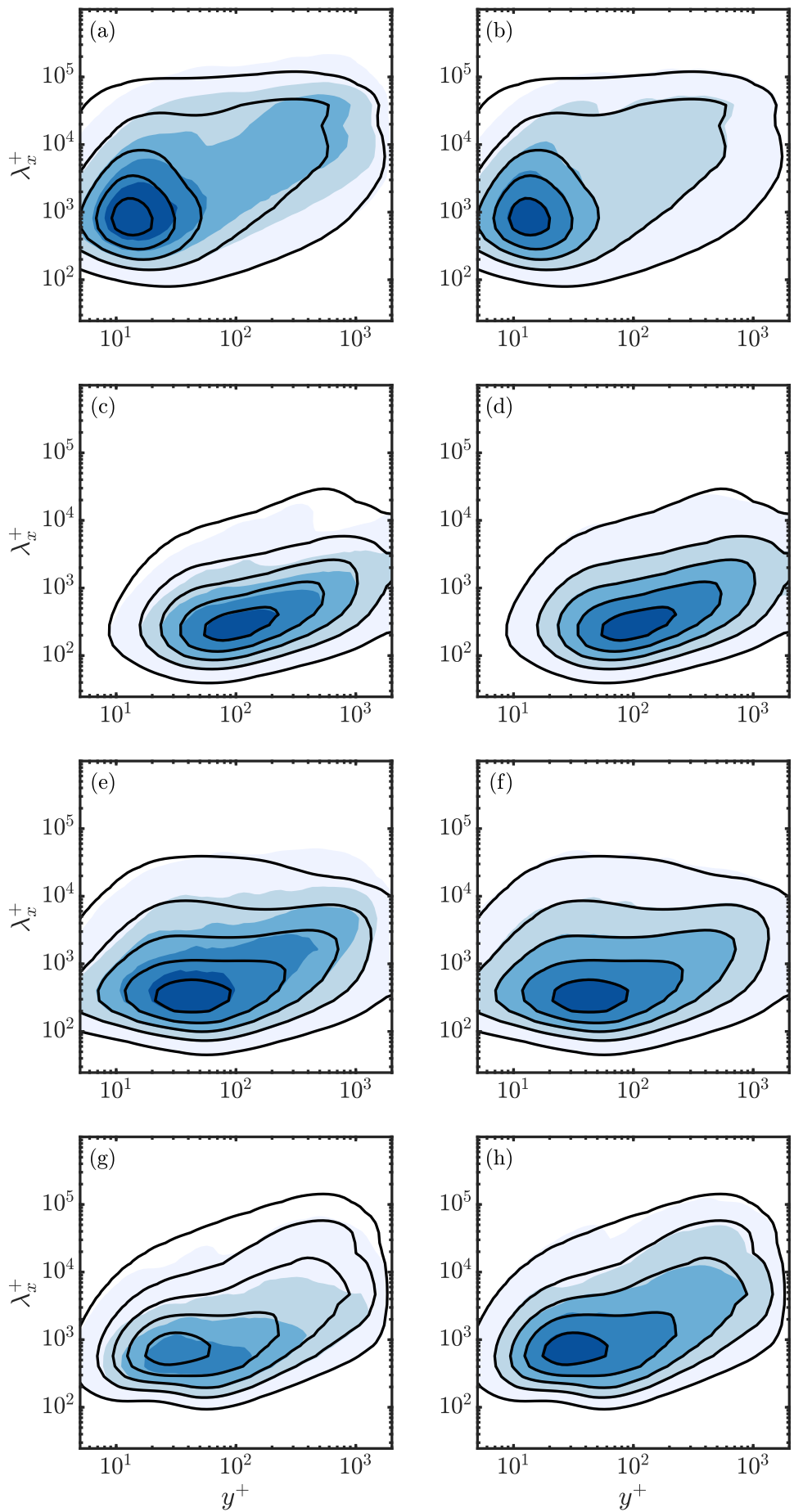

FIG. 1. Premultiplied one-dimensional spectra from the resolvent (filled contours) and DNS (black contours) for $\mathrm{Re}_{\tau}=2003$. (a), (c), (e), (g) Standard resolvent decomposition using $N=6$ modes per wavenumber-frequency triplet. (b), (d), (f), (h) OS-SQ resolvent decomposition using $N^{\mathrm{OS}}=N^{\mathrm{SQ}}=3$ modes per wave-number-frequency triplet. (a), (b) $k_{x} E_{u u}$. (c), (d) $k_{x} E_{v v}$. (e), (f) $k_{x} E_{w w}$. (g), (h) $-k_{x} E_{u v}$. Contour levels are from 10 to $90 \%$ of the DNS maximum in $20 \%$ increments. 

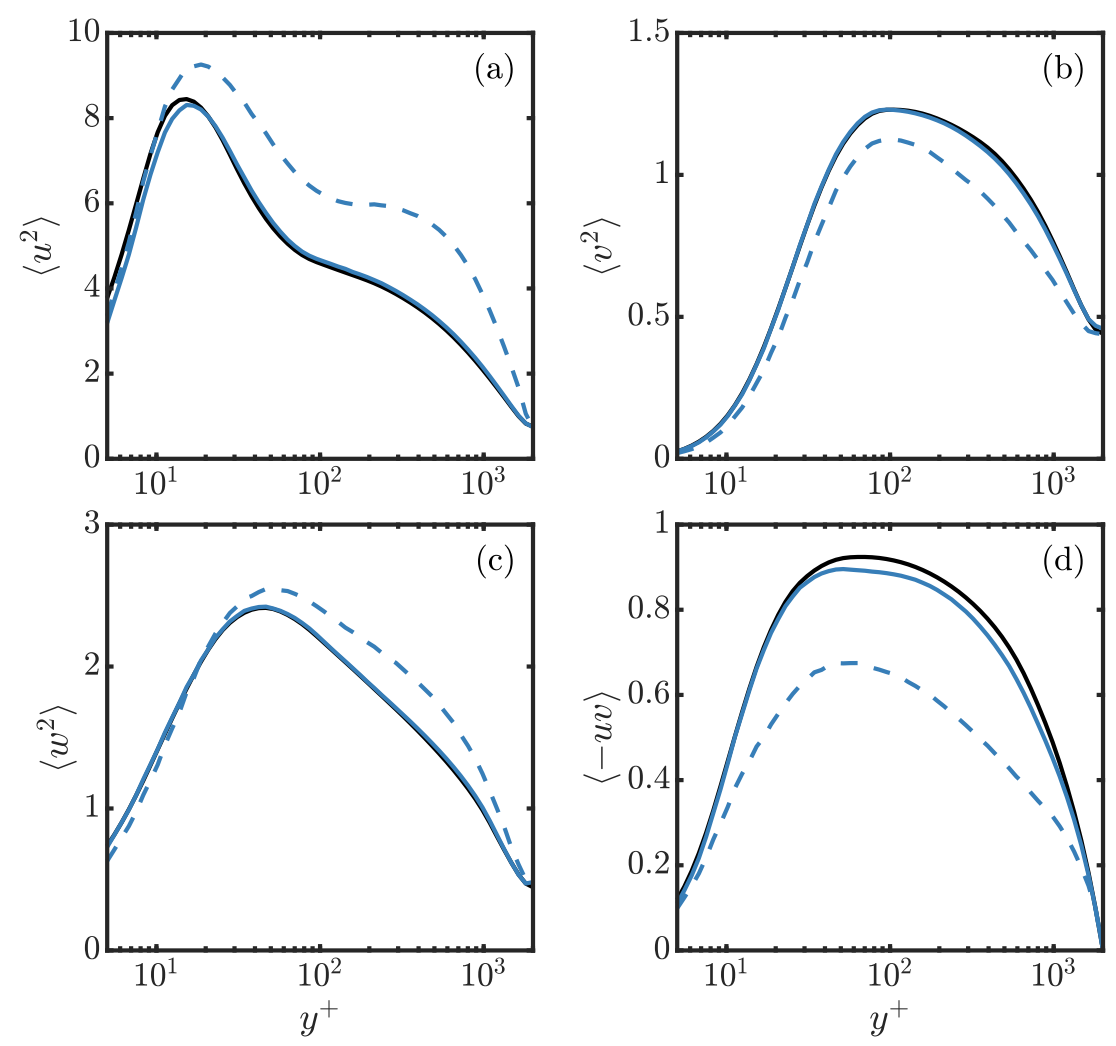

FIG. 2. Intensities from the resolvent with $N^{\mathrm{OS}}=N^{\mathrm{SQ}}=3$ modes per wave-number-frequency triplet (blue) and DNS (black) for $\operatorname{Re}_{\tau}=2003$. Also shown in dashed lines are the intensities obtained from the standard resolvent decomposition approach using the same total number of modes.

and $y^{+} \lesssim 50$. Further discussion of these discrepancies, as well as of the accuracy of the optimized spectra using different numbers of modes, is given in Appendix A. Also shown in the left column are the spectra obtained using the standard decomposition with the same total number of modes. The performance is significantly worse, with $k_{x} E_{u u}$ and $k_{x} E_{w w}$ being greatly overpredicted, and $k_{x} E_{v v}$ and $-k_{x} E_{u v}$ being underpredicted. In fact, the standard resolvent decomposition fails to capture the $90 \%$ energy level (darkest blue contours) for $-k_{x} E_{u v}$. Subsequent integration over $k_{x}$ gives the intensities, which are shown in Fig. 2. The deviation errors are 4.3, 0.95, 0.66, and 3.8\% for $\left\langle u^{2}\right\rangle$, $\left\langle v^{2}\right\rangle,\left\langle w^{2}\right\rangle$, and $\langle-u v\rangle$, respectively. These should be compared with errors of $30,14,12$, and $31 \%$ using the standard resolvent decomposition, shown in the dashed curves.

As the goal of the optimized spectra is to obtain a low-order representation of the spectra, it is worth comparing the number of degrees of freedom of the resolvent spectra to the original DNS. For a given $k_{x}, k_{z}$, the $\operatorname{Re}_{\tau}=2003$ DNS spectra were computed using $N_{y}=635$ wall-normal grid points and $N_{t}=7730$ snapshots [19]. For the results shown in Figs. 1 and 2, the resolvent representation was computed using $N=6$ resolvent modes and $N_{c}=100$ wave speeds, about $0.01 \%$ of the degrees of freedom in the DNS.

\section{B. Prediction of additional statistics}

Since the optimization only attempts to match time-averaged spectra, the distribution of energetic content in $c$ is not directly constrained. To assess this, the power spectra computed from the resolvent representation using the optimized weights with $N^{\mathrm{OS}}=N^{\mathrm{SQ}}=3$ are compared to those computed 

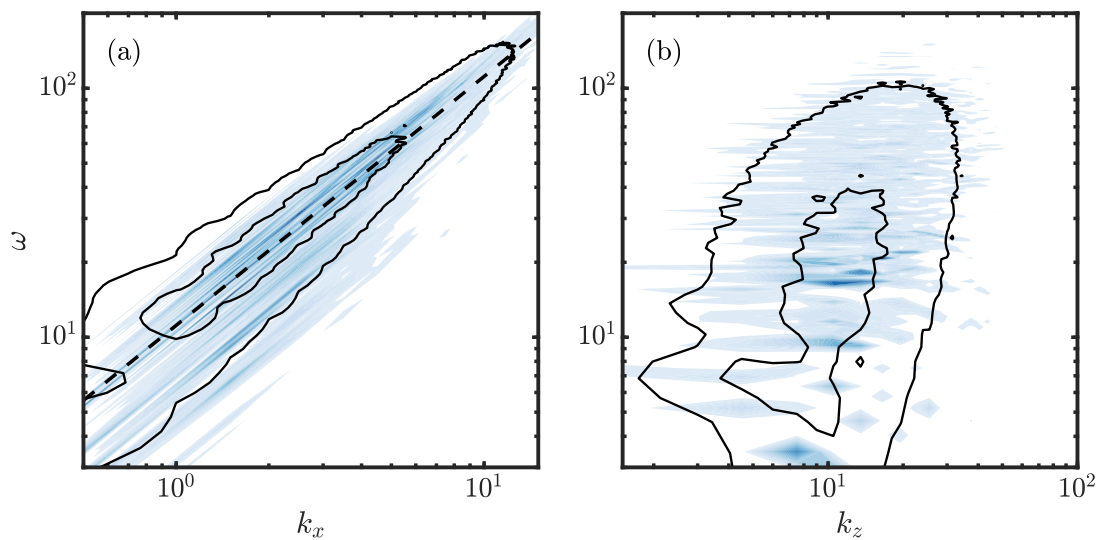

FIG. 3. 2D premultiplied streamwise velocity power spectra at $y^{+} \approx 15$ for $\operatorname{Re}_{\tau}=185$. (a) $\omega k_{x} E_{u u}$. (b) $\omega k_{z} E_{u u}$. Filled contours: Optimized weights with $N^{\mathrm{OS}}=N^{\mathrm{SQ}}=3$. Line contours: DNS; levels are 10 and $50 \%$ of the maximum value. The slope of the dashed line indicates the the local mean velocity $U\left(y^{+}=15\right)$.

from DNS for $\mathrm{Re}_{\tau}=185$ using Welch's method with 8491 snapshots divided into ten segments having $50 \%$ overlap. Figures 3(a) and 3(b) show the 2D premultiplied streamwise power spectra in the $k_{x}-\omega$ and $k_{z}-\omega$ planes, respectively, at $y^{+} \approx 15$.

The distribution in the $k_{x}-\omega$ plane is fairly good, with most of the energetic content of the resolvent spectrum falling within the $10 \%$ DNS contour. As discussed above, the distribution in $c$ is not directly constrained. However, the localization of the leading resolvent modes at the critical layer implies that the energetic content at a given wall-normal location is largely contributed by modes with a wave speed matching the local mean velocity. This is evident in Fig. 3, where both the DNS and resolvent spectra are concentrated around the dashed line representing a constant wave speed $c=U\left(y^{+}=15\right)$. There is no such localization mechanism in the $k_{z}-\omega$ plane. Nonetheless, the resolvent representation still reproduces the general shape of the DNS spectrum quite well. Note that to produce Fig. 3(b) the resolvent spectrum was interpolated onto a common $\omega$ grid prior to integration over $k_{x}$.

The optimized weights can also be used to compute an approximation of the forcing spectra in a manner that is directly analogous to the velocity spectra in Eq. (16):

$$
\begin{aligned}
& E_{g_{v} g_{v}}\left(y, k_{x}, k_{z}, c\right)=\operatorname{Re}\left\{\operatorname{tr}\left(\boldsymbol{B}_{v v} \boldsymbol{X}^{\mathrm{OS} / \mathrm{OS}}\right)\right\}, \\
& E_{g_{\eta} g_{\eta}}\left(y, k_{x}, k_{z}, c\right)=\operatorname{Re}\left\{\operatorname{tr}\left(\boldsymbol{B}_{\eta \eta} \boldsymbol{X}^{\mathrm{SQ} / \mathrm{SQ}}\right)\right\},
\end{aligned}
$$

where $B_{v v, i j}=\phi_{v, i} \phi_{v, j}^{*}$ and $B_{\eta \eta, i j}=\phi_{\eta, i} \phi_{\eta, j}^{*}$. The estimates of the 2D forcing spectra with $N^{\mathrm{OS}}=$ $N^{\mathrm{SQ}}=3$ in the $k_{x}-k_{z}$ plane at $y^{+} \approx 15$ for $\operatorname{Re}_{\tau}=185$ are compared to the full forcing spectra computed from DNS [23] in Fig. 4. The resolvent estimate reasonably predicts the general shape of the full spectra with only a few modes; this is consistent with results indicating that the OS-SQ decomposition yields not only an efficient response basis, but also a forcing basis that is more efficient than the one obtained from the standard resolvent approach [22]. Furthermore, this estimate was obtained using only information about the velocity statistics, an interesting implication of which is that potentially much can be be learned about the nonlinear forcing directly from commonly computed flow quantities. We note that similar observations have been made by Towne et al. [10], who use a limited set of flow statistics to infer forcing statistics, which are in turn used to estimate the unknown flow statistics.

To give additional insight into how energetic modes are distributed across spectral space, the magnitudes of the total mode coefficients, i.e., the weight multiplied by the singular value, are plotted for the leading OS and SQ modes in Figs. 5(a) and 5(b), respectively, for $\operatorname{Re}_{\tau}=2003$; 

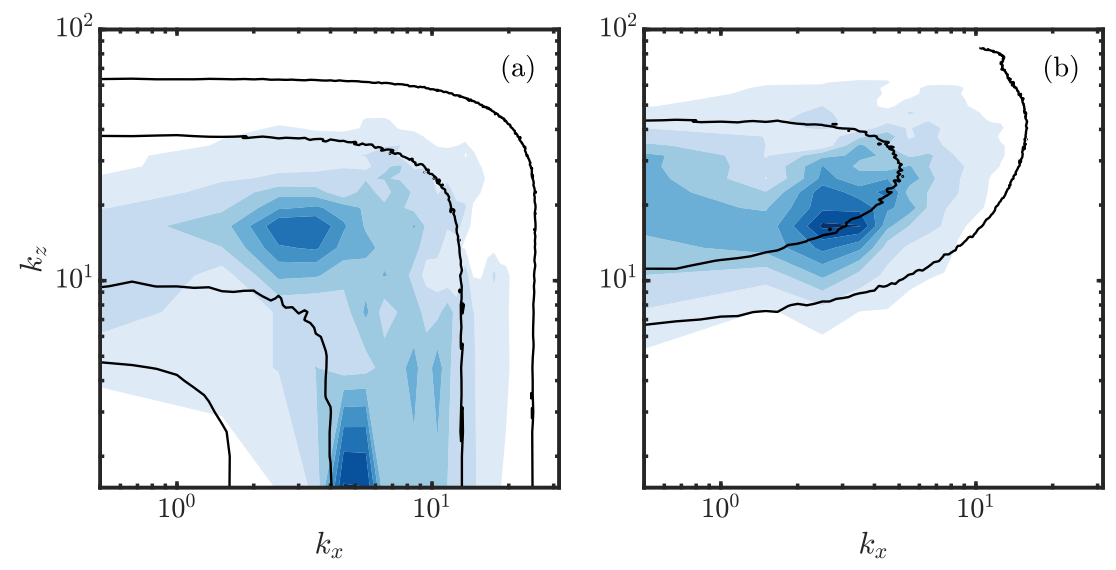

FIG. 4. 2D forcing spectra at $y^{+} \approx 15$ for $\operatorname{Re}_{\tau}=185$. (a) $E_{g_{v} g_{v}}$. (b) $E_{g_{\eta} g_{\eta}}$. Filled contours: Optimized weights with $N^{\mathrm{OS}}=N^{\mathrm{SQ}}=3$. Line contours, reproduced from Rosenberg [23]: DNS; levels are 10 and $50 \%$ of the maximum value.

for ease of visualization, only coefficients larger than $1 \%$ of the maximum value over all spectral space are plotted. Interestingly, they are largely concentrated at large $\lambda_{x}$ and $c$ close to $U_{\mathrm{CL}}$. In addition, there are large coefficients for very low $c$ and large $\lambda_{x}^{+}$, which are likely related to near-wall overcompensation, discussed in Appendix A. This observed clustering may have implications for further model reduction by highlighting important regions of spectral space. With the exception of some SQ coefficients at small $\lambda_{x}^{+}$and $c \approx U_{\mathrm{CL}}$, the large OS and SQ coefficients occupy essentially the same regions of spectral space, which is a reflection of the interactions between the two families of modes; this is discussed in detail in the next section.

Finally, while Fig. 5 appears to suggest that the longest modes should dominate the spectra, we remark that since modes with very large $\lambda_{x}$ typically span the entire channel the orthonormality constraint requires that their peak amplitudes be relatively low compared to other modes having more compact wall-normal support. Thus their contribution to the spectra at a given wall-normal location may be comparable to modes having smaller coefficients.
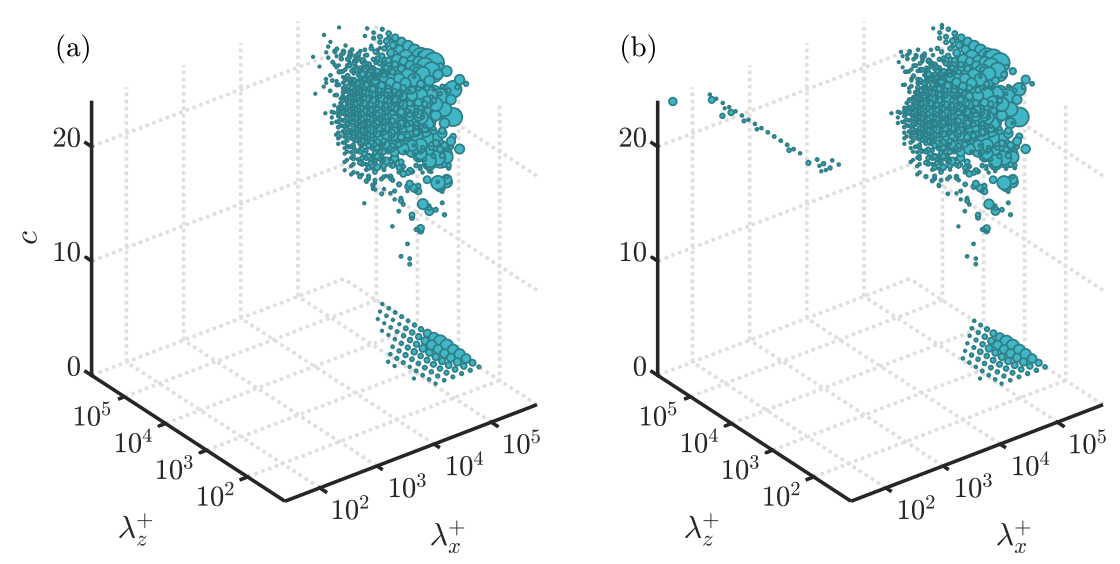

FIG. 5. Magnitudes of the total leading mode coefficients (a) $\sigma_{1}^{\mathrm{OS}} \sqrt{X_{11}^{\mathrm{OS} / \mathrm{OS}}}$ and (b) $\sigma_{1}^{\mathrm{SQ}} \sqrt{X_{11}^{\mathrm{SQ} / \mathrm{SQ}}}$ larger than $1 \%$ of the maximum value over all of spectral space for $\operatorname{Re}_{\tau}=2003$. Marker sizes are proportional to the magnitude and are normalized by the maximum. The axes show the full range of wave parameters included in the optimization. 


\section{Interpretation of the OS-SQ decomposition: A competition mechanism}

It has been demonstrated that the performance of the optimization is greatly improved by employing the OS-SQ decomposition of the resolvent. Previous work reported similar results for channel ECS [22,23]. In that case, the relatively poor performance of the traditional resolvent method was attributed to the fact that the $\eta$ response dominates under the kinetic-energy norm. Thus, matching the statistics for $u$ (or $w$ ) results in underprediction of the $v$ statistics, as observed in Figs. 1 and 2. However, in the OS-SQ decomposition, isolating the $v$ response in only the OS modes allows $v$ and $\eta$ to be "tuned" somewhat independently, with the role of the SQ modes then being to saturate the OS vorticity. The improved matching of all components in Fig. 2 indicates that this is also the case for fully turbulent channel flow, where the dynamics are significantly more complex than for the aforementioned equilibria.

To understand why the OS and SQ modes comprise a much more efficient basis, note that in certain cases the response modes of the standard resolvent, Eq. (12), coincide with those of the OS resolvent, Eq. (14a). A detailed description of the regions of parameter space where this holds is beyond the present scope, but we note that since $\mathcal{H}_{\eta v}$ contains the coupling term $-i k_{z} U^{\prime}$ it is expected to hold whenever the lift-up mechanism is dominant, one such example being for highly streamwise-elongated modes. Further discussion can be found in Dawson and McKeon [36]. Here, we simply illustrate by example for a particular wave-number-frequency triplet in Fig. 6, which compares the singular values and magnitudes of the $\hat{\eta}$ response for the standard, OS, and SQ resolvents. Due to the symmetry of the channel geometry about $y=0$, the resolvent yields symmetric-antisymmetric pairs of modes. If the modes are localized away from the centerline, then their singular values are equal; if, however, the modes have nonzero support at the centerline, then the singular values are not equal but are close in magnitude. The singular values of the OS and standard resolvents are almost equal, with the separation between them growing slowly with increasing mode index. Looking now at the $\hat{\eta}$ response modes, those from OS and standard resolvents are almost indistinguishable. Though not shown, the same is true for the $\hat{v}$ responses.

The SQ singular values are significantly smaller than those for the standard or OS resolvent-by more than an order of magnitude for the first pair. Interestingly, the SQ singular values do not demonstrate clear pairing beyond this. The SQ $\hat{\eta}$ modes are distinct from the other two, in particular having slightly narrower wall-normal support. However, the shapes are still largely similar. Importantly, there is still a significant region of overlap with the OS modes in the wallnormal direction, which is a necessary condition for the SQ modes to interact with the OS modes.

It is also instructive to look at the corresponding forcing modes, which are shown in the top row of Fig. 7. As with the response modes, $\phi_{v}$ for the OS and standard resolvents are virtually identical. This is at first surprising since the standard resolvent has $\phi_{\eta}$ with comparable amplitude to $\phi_{v}$. However, its contribution to the norm is $\lesssim 1 \%$. The bottom row of Fig. 7 shows $\phi_{\eta}$ for the SQ and standard resolvents normalized by their maximum amplitude for ease of comparison. Despite some differences that become more pronounced for the higher-order modes, their shapes are overall quite similar. Therefore, though there may be traces of the SQ modes in the leading standard resolvent modes, they are clearly dominated by the OS ones. This implies that using the standard resolvent operator to generate a low-order representation is effectively equivalent to using only the OS family of modes, and the linear mechanisms encoded in the SQ operator are thus not accounted for.

To further examine the relationship between the OS and SQ modes, we decompose the intensities shown in Fig. 2 into contributions from OS modes only, SQ modes only, and a cross term (C) that represents the interaction of OS and SQ modes, e.g., $\left\langle u^{2}\right\rangle$ becomes

$$
\left\langle u^{2}\right\rangle=\underbrace{\left\langle\left(u^{\mathrm{OS}}\right)^{2}\right\rangle}_{\mathrm{OS}}+\underbrace{\left\langle\left(u^{\mathrm{SQ}}\right)^{2}\right\rangle}_{\mathrm{SQ}}+\underbrace{2\left\langle u^{\mathrm{OS}} u^{\mathrm{SQ}}\right\rangle}_{\mathrm{C}} .
$$

The results with $N=3$ for $\left\langle u^{2}\right\rangle,\left\langle w^{2}\right\rangle$, and $\langle-u v\rangle$ are shown in Fig. 8. The decomposition for $\left\langle v^{2}\right\rangle$ is not shown since, as seen from Eq. (14b), the SQ modes have no $v$ response, and hence 

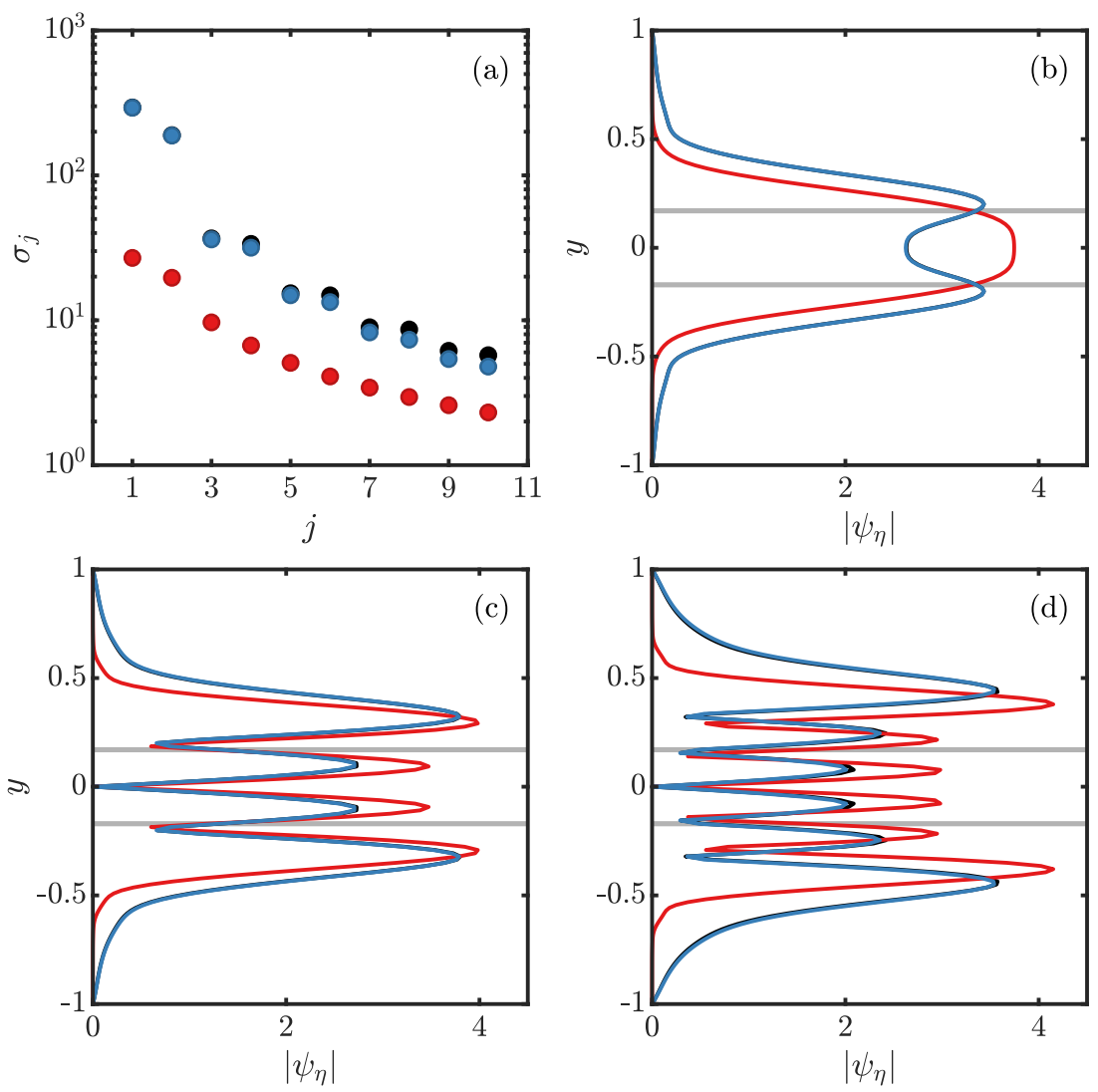

FIG. 6. (a) First ten singular values of the OS (blue), SQ (red), and standard (black) resolvent operators for $\left(k_{x}, k_{z}, c\right)=(0.25,2.5,24.044)$ and $\mathrm{Re}_{\tau}=2003$. (b)-(d) Magnitudes of the vorticity responses from the first, second, and third mode pairs [same color scheme as in (a)]; modes having the same wall-normal symmetry have been selected from each pair. The standard resolvent (black) and OS (blue) modes are visually indistinguishable. The gray lines in (b)-(d) are the locations of the critical layers, $y_{c}= \pm 0.170$.

$\left\langle v^{2}\right\rangle=\left\langle\left(v^{\mathrm{OS}}\right)^{2}\right\rangle$. Similarly, there is no SQ-only contribution to $\langle-u v\rangle$. For $\left\langle u^{2}\right\rangle$ and $\left\langle w^{2}\right\rangle$, the OS and SQ terms are similar, with the OS term having slightly larger magnitude. However, for all three components the $\mathrm{C}$ term is negative, which supports the claim that the SQ vorticity acts to saturate the OS vorticity. In fact, information about the phase relationship between the OS and SQ modes can be deduced from this observation. Note that the third term in Eq. (24) is simply twice the covariance of $u^{\mathrm{OS}}$ and $u^{\mathrm{SQ}}$. For simplicity, express each as a Fourier sine series in one variable:

$$
\begin{aligned}
& u^{\mathrm{OS}}=\sum_{i=1}^{\infty} A_{i}^{\mathrm{OS}} \sin \left(k_{i} x+\theta_{i}^{\mathrm{OS}}\right), \\
& u^{\mathrm{SQ}}=\sum_{i=1}^{\infty} A_{i}^{\mathrm{SQ}} \sin \left(k_{i} x+\theta_{i}^{\mathrm{SQ}}\right),
\end{aligned}
$$



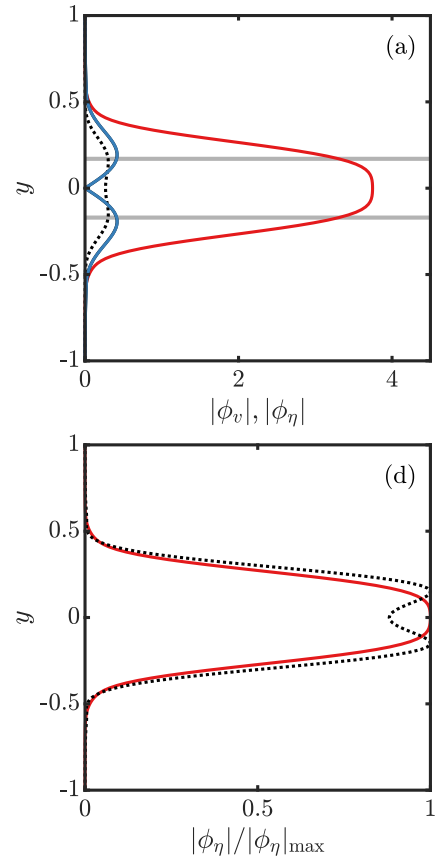
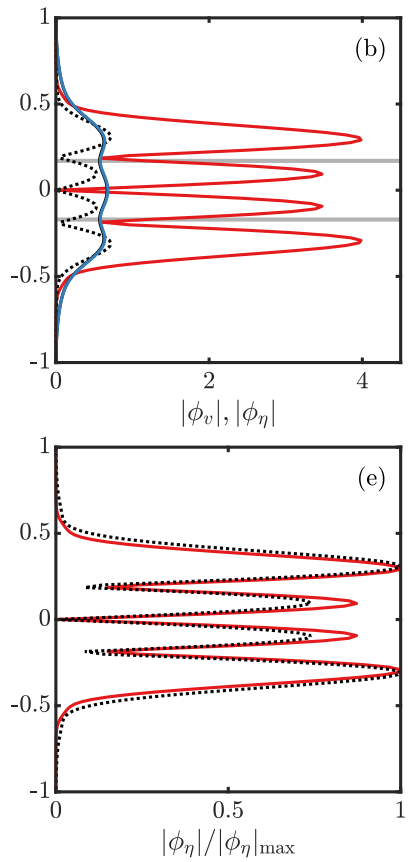
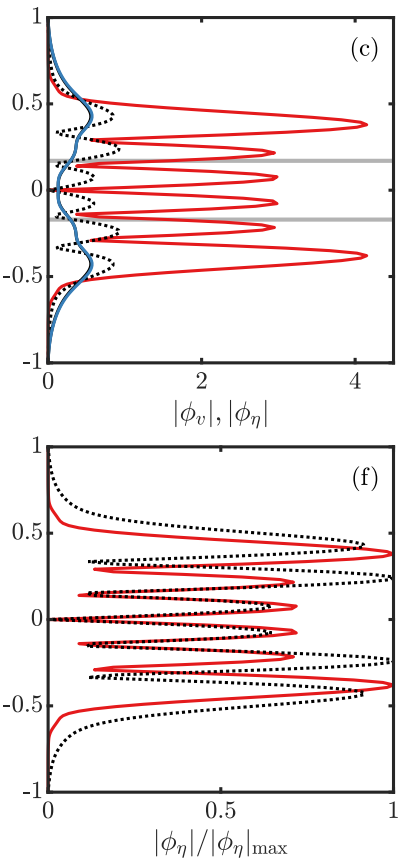

FIG. 7. (a)-(c) Magnitudes of the forcing modes corresponding to the response modes shown in Fig. 6, using the same color scheme. $\phi_{v}$ for the standard resolvent (solid black) is indistinguishable from OS (blue). Dotted lines are the standard resolvent $\phi_{\eta}$. (d)-(f) SQ and standard resolvent $\phi_{\eta}$ normalized by their maximum values. The gray lines in (a)-(c) are the locations of the critical layers, $y_{c}= \pm 0.170$.

where $A_{i}^{\mathrm{X}}$ and $\theta_{i}^{\mathrm{X}}$ are the amplitude and phase of the $i$ th mode, respectively, and $0<k_{1}<k_{2}<\cdots$. Then,

$$
\begin{aligned}
2\left\langle u^{\mathrm{OS}} u^{\mathrm{SQ}}\right\rangle & =2 \sum_{i} \sum_{j} A_{i}^{\mathrm{OS}} A_{j}^{\mathrm{SQ}}\left\langle\sin \left(k_{i} x+\theta_{i}^{\mathrm{OS}}\right) \sin \left(k_{j} x+\theta_{j}^{\mathrm{SQ}}\right)\right\rangle \\
& =\sum_{i} \sum_{j} A_{i}^{\mathrm{OS}} A_{j}^{\mathrm{SQ}}\left(\left\langle\cos \left[\left(k_{i}-k_{j}\right) x+\theta_{i}^{\mathrm{OS}}-\theta_{j}^{\mathrm{SQ}}\right]\right\rangle-\left\langle\cos \left[\left(k_{i}+k_{j}\right) x+\theta_{i}^{\mathrm{OS}}+\theta_{j}^{\mathrm{SQ}}\right]\right\rangle\right) \\
& =\sum_{i} A_{i}^{\mathrm{OS}} A_{i}^{\mathrm{SQ}} \cos \left(\Delta \theta_{i}\right)
\end{aligned}
$$
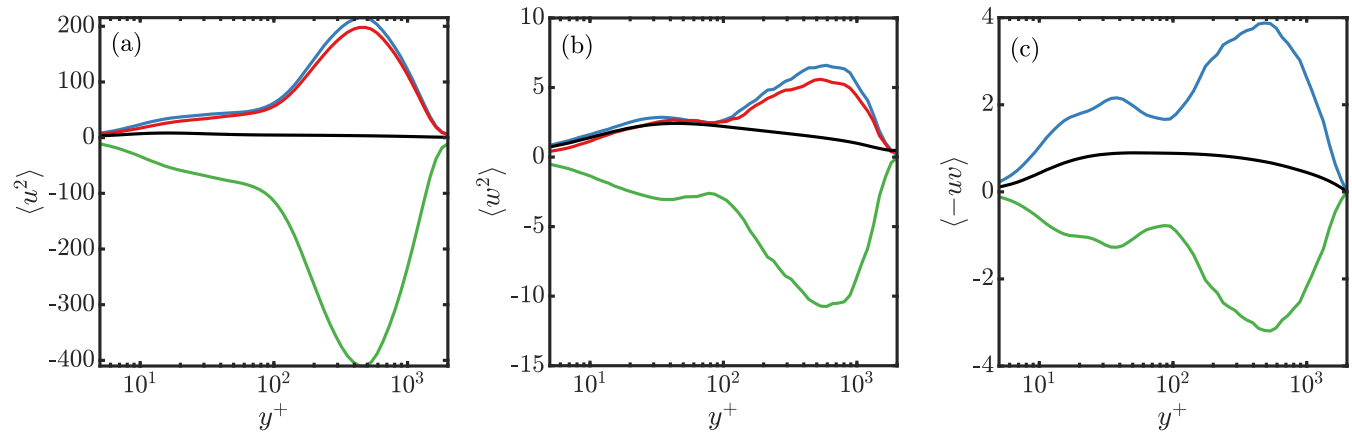

FIG. 8. (a) $\left\langle u^{2}\right\rangle$, (b) $\left\langle w^{2}\right\rangle$, and (c) $\langle-u v\rangle$ decomposed into OS (blue), SQ (red), and C (green) terms for $N=3$. The totals are plotted in black. 

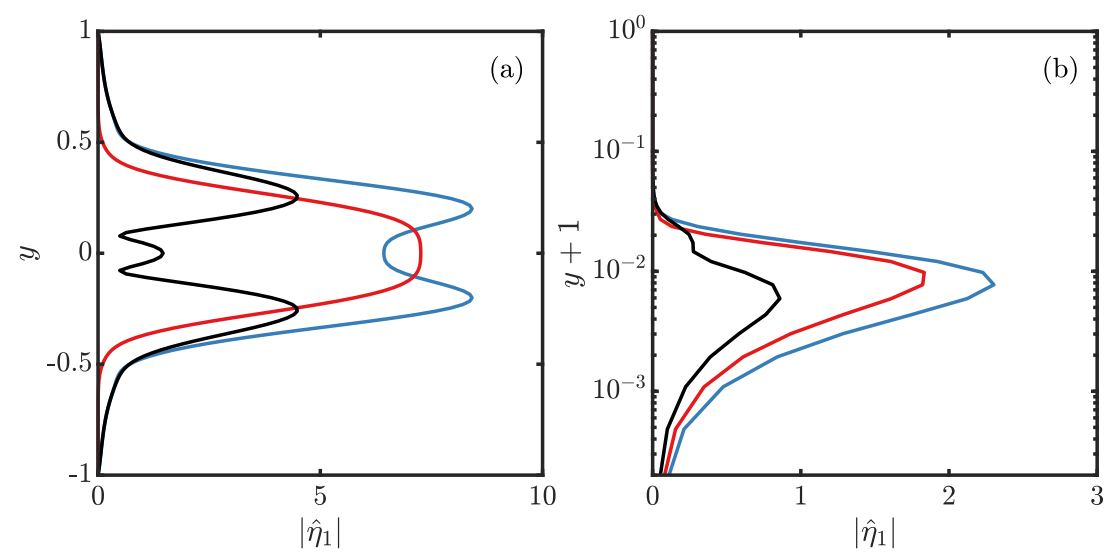

FIG. 9. Magnitudes of the leading vorticity response: $\left|\hat{\eta}_{1}^{\mathrm{OS}}\right|$ (blue), $\left|\hat{\eta}_{1}^{\mathrm{SQ}}\right|$ (red), and $\left|\hat{\eta}_{1}^{\mathrm{OS}}+\hat{\eta}_{1}^{\mathrm{SQ}}\right|$ (black). (a) $\left(k_{x}, k_{z}, c\right)=(0.25,2.5,24.044)$. (b) $\left(k_{x}, k_{z}, c\right)=(10,100,10.059)$. The modes in (b) are plotted against $y+1$ due to the log scale.

where $\Delta \theta_{i}=\theta_{i}^{\mathrm{OS}}-\theta_{i}^{\mathrm{SQ}}$. The term labeled C in Eq. (24) can thus be interpreted as a weighted (by the amplitudes) sum of the cosines of the phase difference between the OS and SQ modes. Therefore, $\left\langle u^{\mathrm{OS}} u^{\mathrm{SQ}}\right\rangle<0$ implies $\pi / 2<\left|\Delta \theta_{i}\right|<3 \pi / 2$ on average. Furthermore, the relative magnitudes of the three terms suggest that for the majority of modes the phase difference is relatively close to $\pi$, i.e., the OS and SQ modes are close to being exactly out of phase. Finally, we note that while the individual terms in Eq. (24) depend on $N$, the trends discussed above, namely, the similarity of the OS and SQ terms and the $\mathrm{C}$ term being negative, do not. Furthermore, performing the decomposition in Eq. (24) for $\left\langle\eta^{2}\right\rangle$ from $\operatorname{Re}_{\tau}=185$ DNS data reveals the same features [23]. This provides strong evidence that they are not merely consequences of the particular optimization procedure, but are in fact robust features of turbulent channel flow.

Since the SQ modes are exclusively wall-parallel motions, there is a passing resemblance to the notion of the "inactive" motions proposed by Townsend [37]. It is supposed that, at first order, the inactive motions do not interact with the "active" shear stress-carrying motions. However, Fig. 8(c) shows that the interaction of the SQ vorticity with $v$ produced by the OS modes, the C term, contributes significantly to the overall Reynolds shear stress profile, suggesting that there is not an exact correspondence between the SQ modes and inactive motions.

To better understand how the competition between the OS and SQ vorticities manifests itself on a mode-by-mode basis, we compute the sum of the leading OS and SQ vorticity modes, including their singular values and resolvent weights, that is, the first term from each sum in Eq. (15):

$$
\begin{aligned}
\hat{\eta}_{1}^{\mathrm{OS}}+\hat{\eta}_{1}^{\mathrm{SQ}} & =\psi_{\eta, 1}^{\mathrm{OS}} \sigma_{1}^{\mathrm{OS}} \chi_{1}^{\mathrm{OS}}+\psi_{\eta, 1}^{\mathrm{SQ}} \sigma_{1}^{\mathrm{SQ}} \chi_{1}^{\mathrm{SQ}} \\
& =\psi_{\eta, 1}^{\mathrm{OS}} \sigma_{1}^{\mathrm{OS}}\left|\chi_{1}^{\mathrm{OS}}\right| \mathrm{e}^{i \theta_{1}^{\mathrm{OS}}}+\psi_{\eta, 1}^{\mathrm{SQ}} \sigma_{1}^{\mathrm{SQ}}\left|\chi_{1}^{\mathrm{SQ}}\right| \mathrm{e}^{i \theta_{1}^{\mathrm{SQ}}} \\
& =\left(\psi_{\eta, 1}^{\mathrm{OS}} \sigma_{1}^{\mathrm{OS}}\left|\chi_{1}^{\mathrm{OS}}\right|+\psi_{\eta, 1}^{\mathrm{SQ}} \sigma_{1}^{\mathrm{SQ}}\left|\chi_{1}^{\mathrm{SQ}}\right| \mathrm{e}^{-i \Delta \theta_{1}}\right) \mathrm{e}^{i \theta_{1}^{\mathrm{OS}}}
\end{aligned}
$$

where $\Delta \theta_{1}$ is defined as in Eq. (26). Though the absolute phase, i.e., $\theta_{1}^{\mathrm{OS}}$, cannot be determined from the optimization, all quantities inside the parentheses can be. From Eq. (19), we have

$$
\left|\chi_{1}^{\mathrm{OS}}\right|=\sqrt{X_{11}^{\mathrm{OS} / \mathrm{OS}}}, \quad\left|\chi_{1}^{\mathrm{SQ}}\right|=\sqrt{X_{11}^{\mathrm{SQ} / \mathrm{SQ}}}, \quad-\Delta \theta_{1}=\arg \boldsymbol{X}_{11}^{\mathrm{OS} / \mathrm{SQ}},
$$

where arg denotes the argument of a complex number. The magnitude of Eq. (27) is plotted for two different wave-number-frequency triplets in Fig. 9, along with the magnitudes of the OS and SQ vorticities. Figure 9(a) shows the same mode plotted in Fig. 6, and Fig. 9(b) shows a 

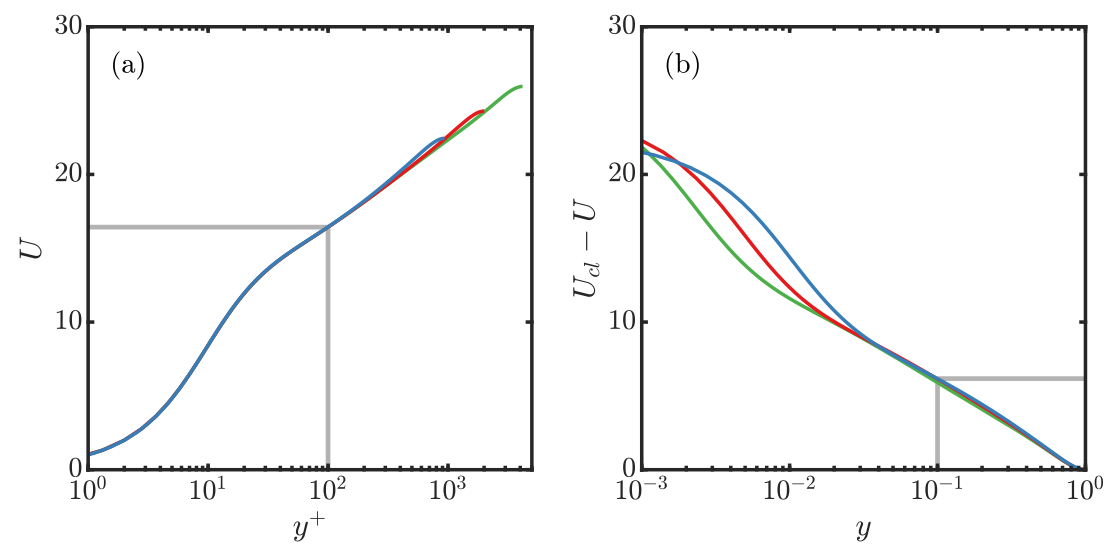

FIG. 10. (a) Mean velocity profile $U\left(y^{+}\right)$and (b) velocity defect $U_{\mathrm{CL}}-U(y)$ for $\operatorname{Re}_{\tau}=934$ (blue), $\operatorname{Re}_{\tau}=$ 2003 (red), and $\operatorname{Re}_{\tau}=4219$ (green). The gray boxes indicate the regions where the profiles are $\operatorname{Re}_{\tau}$ invariant in the respective coordinates.

smaller-scale wall-localized mode. In both cases, the sum of the OS and SQ has a significantly smaller amplitude, indicating that they interfere in a destructive manner. This is consistent with the conclusions drawn from Fig. 8 and demonstrates that this competition mechanism is active over a broad range of spatiotemporal scales. Further, in each case the sum of the OS and SQ modes has a larger relative support in $y$ for a given percentage of the peak value than for either individual mode, which is likely to be part of the reason behind the efficient representation associated with the OS-SQ decomposition.

In this section it was shown that the OS-SQ decomposition of the resolvent provides an improved basis for efficiently representing the statistics of turbulent channel flow, and that this provides insight into the complex physics at play, namely, a competition mechanism, interpreted as a phase difference, between OS and SQ modes that results in saturation of the wall-normal vorticity. In the next section, we use this insight to derive simple scalings for the relative magnitudes of the OS and SQ weights of modes belonging to several special classes.

\section{WEIGHTS SCALING FOR THE UNIVERSAL CLASSES OF RESOLVENT MODES}

Moarref et al. [25] leveraged universal scaling regimes of the mean velocity profile to derive the $\operatorname{Re}_{\tau}$ scaling for several universal classes of resolvent modes. Here, we extend this to the OS-SQ resolvent decomposition and show that for the outer and geometrically self-similar classes each family of modes has a distinct scaling for the singular values. From this, the scalings of each submatrix of the energy density matrices $\boldsymbol{A}_{r}$ given in Eq. (19) can be determined. Combining these scalings with the hypothesis that competition of the OS and SQ modes remains relevant at different Reynolds numbers and in different regions of the flow enables the relative scaling of the OS and SQ weights belonging to the universal classes to be deduced.

The universal classes investigated here are the inner, outer, and geometrically self-similar classes. These consist of resolvent modes that are localized within the near-wall, wake, and logarithmic regions of the flow, respectively, and rely on universality of the mean velocity profile under the appropriate scaling in these regions. Figure 10(a) demonstrates the universality of $U$ for $y^{+} \lesssim 100$, and Fig. 10(b) shows that the velocity defect $U_{\mathrm{CL}}-U$ is universal for $y \gtrsim 0.1$; these approximate boundaries are indicated by the vertical dashed lines in Fig. 10. Between these regions, there exists an intermediate region of the mean velocity profile in which both scalings hold. In this overlap region, it is widely accepted that the mean varies logarithmically with distance from the wall. Classical estimates put the beginning of the logarithmic region at $y^{+}=O(100)$. However, there 
is recent evidence that this lower boundary moves outward as $\operatorname{Re}_{\tau}^{1 / 2}[38,39]$. In the logarithmic region, the resolvent operator admits self-similar modes localized about their critical layers [25]. Furthermore, the scaling of these modes reduces to the inner and outer scalings when $y^{+}$or $y$, respectively, is held fixed, reflecting their mutual validity in the logarithmic region.

In the next subsections, we briefly summarize the general approach to scaling of the wave parameters as well as the distinct scalings for the OS and SQ singular values for the inner scaling universal class. The relative scaling of the OS and SQ weights is presented and tested against the computed optimal weights for each of the three universal classes derived by Moarref et al. [25].

\section{A. Inner class}

Following Moarref et al. [25], the relevant length scale for the inner class is the viscous unit $v / u_{\tau}$, so that the corresponding inner-scaled parameters are

$$
k_{x}^{+}=\operatorname{Re}_{\tau}^{-1} k_{x}, \quad k_{z}^{+}=\operatorname{Re}_{\tau}^{-1} k_{z}, \quad y^{+}=\operatorname{Re}_{\tau} y,
$$

where a superscript $(\cdot)^{+}$indicates a quantity that is $\operatorname{Re}_{\tau}$ invariant for modes belonging to the inner class. The inner class wave parameters are

$$
\mathscr{S}_{i}: \quad 0 \leqslant c \lesssim 16.4
$$

The upper wave-speed limit is obtained from the critical layer at the top of the inner region, i.e., $U\left(y^{+}=100\right)=16.4$; this is indicated by the horizontal dashed line in Fig. 10(a). Note that this bound is slightly different from the one given in Moarref et al. [25] since the mean velocity profiles they used were obtained from an eddy viscosity model, whereas the ones used here are taken directly from the DNS that the spectra are obtained from. Using Eq. (29) and continuity, it follows that all three velocity components scale in the same way. Furthermore, the orthonormality constraint on the resolvent modes imposes

$$
\hat{\boldsymbol{u}}=\operatorname{Re}_{\tau}^{1 / 2} \hat{\boldsymbol{u}}^{+}
$$

Equation (29) can be used to obtain the inner-scaled versions of the weighted resolvent operators:

$$
\begin{gathered}
\left(\begin{array}{c}
\mathcal{F}_{v} \mathcal{H}_{v v} \mathcal{F}_{v}^{-1} \\
\mathcal{F}_{\eta} \mathcal{H}_{\eta v} \mathcal{F}_{v}^{-1}
\end{array}\right)=\operatorname{Re}_{\tau}^{-1}\left(\begin{array}{l}
\mathcal{F}_{v}^{+} \mathcal{H}_{v v}^{+} \mathcal{F}_{v}^{+-1} \\
\mathcal{F}_{\eta}^{+} \mathcal{H}_{\eta v}^{+} \mathcal{F}_{v}^{+-1}
\end{array}\right), \\
\mathcal{F}_{\eta} \mathcal{H}_{\eta \eta} \mathcal{F}_{\eta}^{-1}=\operatorname{Re}_{\tau}^{-1} \mathcal{F}_{\eta}^{+} \mathcal{H}_{\eta \eta}^{+} \mathcal{F}_{\eta}^{+-1},
\end{gathered}
$$

where $\mathcal{F}=\operatorname{diag}\left(\mathcal{F}_{v}, \mathcal{F}_{\eta}\right)$ is the square root of the positive-definite operator $\mathcal{Q}$ defined in Eq. (9), i.e., $\mathcal{Q}=\mathcal{F}^{\dagger} \mathcal{F}$, and the superscript $(\cdot)^{\dagger}$ denotes the adjoint with respect to the inner product Eq. (8). Computing the SVDs of Eqs. (32a) and (32b), it is clear that both the OS and SQ singular values have the same (Reynolds number) scaling:

$$
\sigma_{j}^{\mathrm{OS}}=\mathrm{Re}_{\tau}^{-1} \sigma_{j}^{\mathrm{OS}+}, \quad \sigma_{j}^{\mathrm{SQ}}=\mathrm{Re}_{\tau}^{-1} \sigma_{j}^{\mathrm{SQ}+} .
$$

An example of the collapse of the scaled OS, SQ, and standard inner class leading vorticity response modes and scaled forcing modes with Reynolds number is given in Appendix B.

Substituting Eqs. (31) and (33) into Eq. (17), we obtain the inner-scaled energy density matrices:

$$
\boldsymbol{A}_{r, i j}^{\mathrm{OS} / \mathrm{OS}}=\mathrm{Re}_{\tau}^{-1} \boldsymbol{A}_{r, i j}^{\mathrm{OS} / \mathrm{OS}+}, \quad \boldsymbol{A}_{r, i j}^{\mathrm{OS} / \mathrm{SQ}}=\mathrm{Re}_{\tau}^{-1} \boldsymbol{A}_{r, i j}^{\mathrm{OS} / \mathrm{SQ}+}, \quad \boldsymbol{A}_{r, i j}^{\mathrm{SQ} / \mathrm{SQ}}=\mathrm{Re}_{\tau}^{-1} \boldsymbol{A}_{r, i j}^{\mathrm{SQ} / \mathrm{SQ}+} .
$$

With this, the decomposed version of the three-dimensional streamwise energy spectrum becomes

$$
\begin{aligned}
E_{u u}= & \operatorname{Re}_{\tau}^{-1} \operatorname{Re}\left\{\operatorname{tr}\left(\boldsymbol{A}_{u u}^{\mathrm{OS} / \mathrm{OS}+} \boldsymbol{X}^{\mathrm{OS} / \mathrm{OS}}\right)\right\}+2 \operatorname{Re}_{\tau}^{-1} \operatorname{Re}\left\{\operatorname{tr}\left(\boldsymbol{A}_{u u}^{\mathrm{SQ} / \mathrm{OS}+} \boldsymbol{X}^{\mathrm{OS} / \mathrm{SQ}}\right)\right\} \\
& +\operatorname{Re}_{\tau}^{-1} \operatorname{Re}\left\{\operatorname{tr}\left(\boldsymbol{A}_{u u}^{\mathrm{SQ} / \mathrm{SQ}+} \boldsymbol{X}^{\mathrm{SQ} / \mathrm{SQ}}\right)\right\},
\end{aligned}
$$



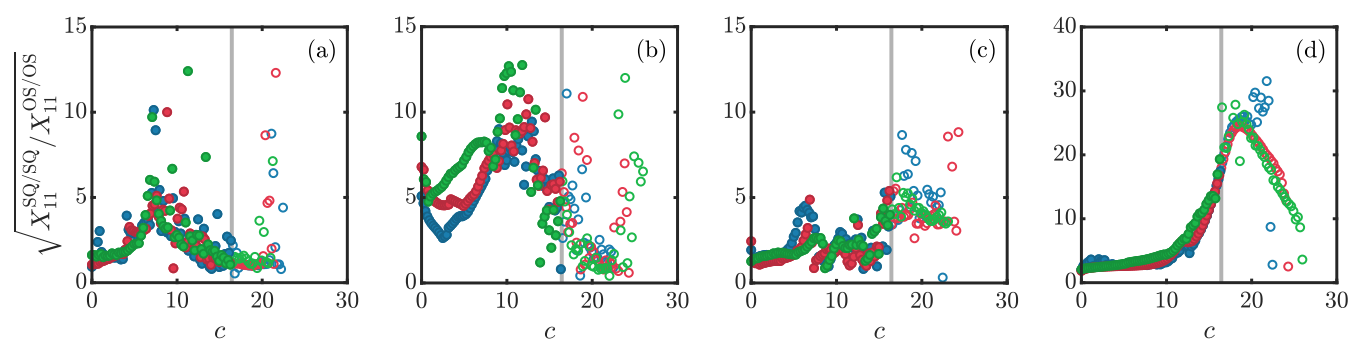

FIG. 11. Leading weights ratio for $\operatorname{Re}_{\tau}=934$ (blue), $\operatorname{Re}_{\tau}=2003$ (red), and $\operatorname{Re}_{\tau}=4219$ (green) with $N=3$ for several different wavelengths: (a) $\left(k_{x}^{+}, k_{z}^{+}\right)=\left(2 \pi / 10^{2}, 2 \pi / 10^{2}\right)$; (b) $\left(k_{x}^{+}, k_{z}^{+}\right)=\left(2 \pi / 10^{3}, 2 \pi / 10^{2}\right)$; (c) $\left(k_{x}^{+}, k_{z}^{+}\right)=\left(2 \pi / 10^{3}, 2 \pi / 10^{3}\right)$; (d) $\left(k_{x}^{+}, k_{z}^{+}\right)=\left(2 \pi / 10^{4}, 2 \pi / 10^{3}\right)$. Filled circles denote modes belonging to the universal inner class, and the highest inner class wave speed, $c=16.4$, is indicated by the gray line.

where the Reynolds number dependence of the right-hand side is made explicit, save for the unscaled weights matrices $\boldsymbol{X}^{\mathrm{X} / \mathrm{Y}}$. Equation (34) can be used to write similar expressions for the other components of the spectra.

Since the overall scaling of $E_{u u}$ for the inner class is not known, the absolute scaling of the weights cannot be determined directly from Eq. (35). However, in Sec. III A it was shown that the vorticities generated by OS and SQ modes compete. That is, the vorticity generated by the SQ modes acts to "saturate" the OS vorticity. We hypothesize that this mechanism is not specific to $\operatorname{Re}_{\tau}=2003$ for which the optimization results were presented, but instead holds for arbitrary $\operatorname{Re}_{\tau}$. This is only possible if all three terms remain of the same order in Eq. (35), which is satisfied if the inner class OS and SQ weights have the same scaling, i.e., if the ratio

$$
\left|\frac{\chi_{j}^{\mathrm{SQ}}}{\chi_{j}^{\mathrm{OS}}}\right| \neq \mathrm{fn}\left(\mathrm{Re}_{\tau}\right)
$$

for modes belonging to the universal inner class.

This scaling is tested by computing the weights matrices for the three Reynolds numbers depicted in Fig. 10 for fixed inner-scaled wave-number combinations $\left(k_{x}^{+}, k_{z}^{+}\right)$. As discussed in Sec. II C, the individual weights are not recovered from the full-rank solutions. However, $\boldsymbol{X}_{j j}^{\mathrm{OS} / \mathrm{OS}} \sim\left|\chi_{j}^{\mathrm{OS}}\right|^{2}$ and $\boldsymbol{X}_{j j}^{\mathrm{SQ} / \mathrm{SQ}} \sim\left|\chi_{j}^{\mathrm{SQ}}\right|^{2}$, so that $\left|\chi_{j}^{\mathrm{SQ}} / \chi_{j}^{\mathrm{OS}}\right| \sim \sqrt{\boldsymbol{X}_{j j}^{\mathrm{SQ} / \mathrm{SQ}} / \boldsymbol{X}_{j j}^{\mathrm{OS} / \mathrm{OS}}}$. This ratio with $j=1$ is computed for the three Reynolds numbers, and the results for several wave-number combinations spanning a large range of scales are shown in Fig. 11. Overall, the agreement is quite good, showing a reasonable collapse despite some scatter. The main exception is for $\left(k_{x}^{+}, k_{z}^{+}\right)=\left(2 \pi / 10^{3}, 2 \pi / 10^{2}\right)$ in Fig. 11(b), where there is clearly some dependence on $\operatorname{Re}_{\tau}$ for $c \lesssim 10$. This trend is consistent for modes of relatively small scale $\left[k_{x}^{+} \gtrsim O\left(2 \pi / 10^{3}\right)\right]$ and large aspect ratio $\left[k_{z}^{+} / k_{x}^{+} \gtrsim O(10)\right]$. The reason for the failure of the scaling for these modes is unclear. However, it is observed that in such cases the profiles of the time-averaged energy spectra are localized very near the wall. Additionally, the upper limit on the wave-speed range for inner class modes given in Eq. (30), $c=16.4$, is shown in each panel of Fig. 11 as the vertical gray line. The fact that the scaling given by Eq. (36) holds reasonably well for $c>16.4$ is a reflection of the fact that the inner scaling remains valid in the logarithmic region, as seen in Fig. 10(a).

\section{B. Outer class}

The outer class length scales are

$$
\tilde{k}_{x}=\operatorname{Re}_{\tau} k_{x}, \quad \tilde{k}_{z}=k_{z}, \quad \tilde{y}=y,
$$


and the outer class wave parameters are

$$
\mathscr{S}_{o}:\left\{\begin{array}{l}
0 \leqslant U_{c l}-c \lesssim 6.17 \\
k_{z} / k_{x} \gtrsim \gamma \operatorname{Re}_{\tau} / \operatorname{Re}_{\tau, \text { min }}
\end{array},\right.
$$

where the upper bound on the wave-speed defect $U_{\mathrm{CL}}-c=6.17$ is obtained from the setting the minimum critical layer location at the bottom of the outer region, i.e., $U_{\mathrm{CL}}-U(y=0.1)=6.17$; this is indicated by the horizontal dashed line in Fig. 10(b). Again, this value is slightly different from the one given in Moarref et al. [25] due to the different source for the mean profiles. As Eq. (38) indicates, the outer class modes must satisfy an aspect ratio constraint for all $\operatorname{Re}_{\tau}$ considered, where the minimum aspect ratio is $\gamma$ when $\operatorname{Re}_{\tau}=\operatorname{Re}_{\tau, \min }$ [25]. Here, $\operatorname{Re}_{\tau, \min }=934$. From Eq. (37) and continuity, it follows that

$$
\hat{\boldsymbol{u}}=\left(\begin{array}{c}
\tilde{u} \\
\operatorname{Re}_{\tau}^{-1} \tilde{v} \\
\operatorname{Re}_{\tau}^{-1} \tilde{w}
\end{array}\right),
$$

where $(\tilde{\cdot})$ indicates a quantity that is approximately $\operatorname{Re}_{\tau}$ invariant for modes belonging to the outer class. See also Sharma et al. [40] and Moarref et al. [41] for the scaling of each velocity component, as well as for the components of the forcing modes.

The outer-scaled versions of the weighted resolvent operators are

$$
\begin{aligned}
\left(\begin{array}{c}
\mathcal{F}_{v} \mathcal{H}_{v v} \mathcal{F}_{v}^{-1} \\
\mathcal{F}_{\eta} \mathcal{H}_{\eta v} \mathcal{F}_{v}^{-1}
\end{array}\right) & =\left(\begin{array}{l}
\operatorname{Re}_{\tau} \tilde{\mathcal{F}}_{v} \tilde{\mathcal{H}}_{v v} \tilde{\mathcal{F}}_{v}^{-1} \\
\operatorname{Re}_{\tau}^{2} \tilde{\mathcal{F}}_{\eta} \tilde{\mathcal{H}}_{\eta v} \tilde{\mathcal{F}}_{v}^{-1}
\end{array}\right), \\
\mathcal{F}_{\eta} \mathcal{H}_{\eta \eta} \mathcal{F}_{\eta}^{-1} & =\operatorname{Re}_{\tau} \tilde{\mathcal{F}}_{\eta} \tilde{\mathcal{H}}_{\eta \eta} \tilde{\mathcal{F}}_{\eta}^{-1} .
\end{aligned}
$$

Computing the SVDs of Eqs. 40a and 40b, we have for the leading singular values

$$
\sigma_{j}^{\mathrm{OS}}=\operatorname{Re}_{\tau}^{2} \tilde{\sigma}_{j}^{\mathrm{OS}}, \quad \sigma_{j}^{\mathrm{SQ}}=\operatorname{Re}_{\tau} \tilde{\sigma}_{j}^{\mathrm{SQ}} .
$$

Note that because the components of Eq. (40a) do not scale uniformly for outer class modes the scaling of the OS singular values is only expected to hold for the first several modes. However, since good agreement between the resolvent and DNS spectra is achieved using only a small number of modes, it is reasonable to adopt the scalings in what follows.

Once again, the collapse of resolvent modes under outer scaling is given in Appendix B; the analysis equivalent to that giving rise to Eq. (34) but for the outer scaling class is also relegated there for clarity of the present development. The latter yields the following expression for the streamwise energy spectrum:

$E_{u u}=\operatorname{Re}_{\tau}^{4} \operatorname{Re}\left\{\operatorname{tr}\left(\tilde{\boldsymbol{A}}_{u u}^{\mathrm{OS} / \mathrm{OS}} \boldsymbol{X}^{\mathrm{OS} / \mathrm{OS}}\right)\right\}+2 \operatorname{Re}_{\tau}^{3} \operatorname{Re}\left\{\operatorname{tr}\left(\tilde{\boldsymbol{A}}_{u u}^{\mathrm{SQ} / \mathrm{OS}} \boldsymbol{X}^{\mathrm{OS} / \mathrm{SQ}}\right)\right\}+\operatorname{Re}_{\tau}^{2} \operatorname{Re}\left\{\operatorname{tr}\left(\tilde{\boldsymbol{A}}_{u u}^{\mathrm{SQ} / \mathrm{SQ}} \boldsymbol{X}^{\mathrm{SQ} / \mathrm{SQ}}\right)\right\}$,

where again the Reynolds number dependence of the right-hand side is explicit, save for the unscaled weights matrices $\boldsymbol{X}^{\mathrm{X} / \mathrm{Y}}$, and Eq. (B1) can be used to write similar expressions for the other energy spectra.

As for the inner class modes, competition between the OS and SQ modes requires that all three terms are of the same order for arbitrary $\mathrm{Re}_{\tau}$, which is satisfied if

$$
\left|\frac{\chi_{j}^{\mathrm{SQ}}}{\chi_{j}^{\mathrm{OS}}}\right| \sim \mathrm{Re}_{\tau}
$$

for modes belonging to the universal outer class.

The weights ratio $\sqrt{X_{11}^{\mathrm{SQ} / \mathrm{SQ}} / X_{11}^{\mathrm{OS} / \mathrm{OS}}}$ for the three Reynolds numbers is shown in the top row of Fig. 12 for several values of the outer-scaled wave-number combinations $\left(\tilde{k}_{x}, \tilde{k}_{x}\right)$ and a minimum 

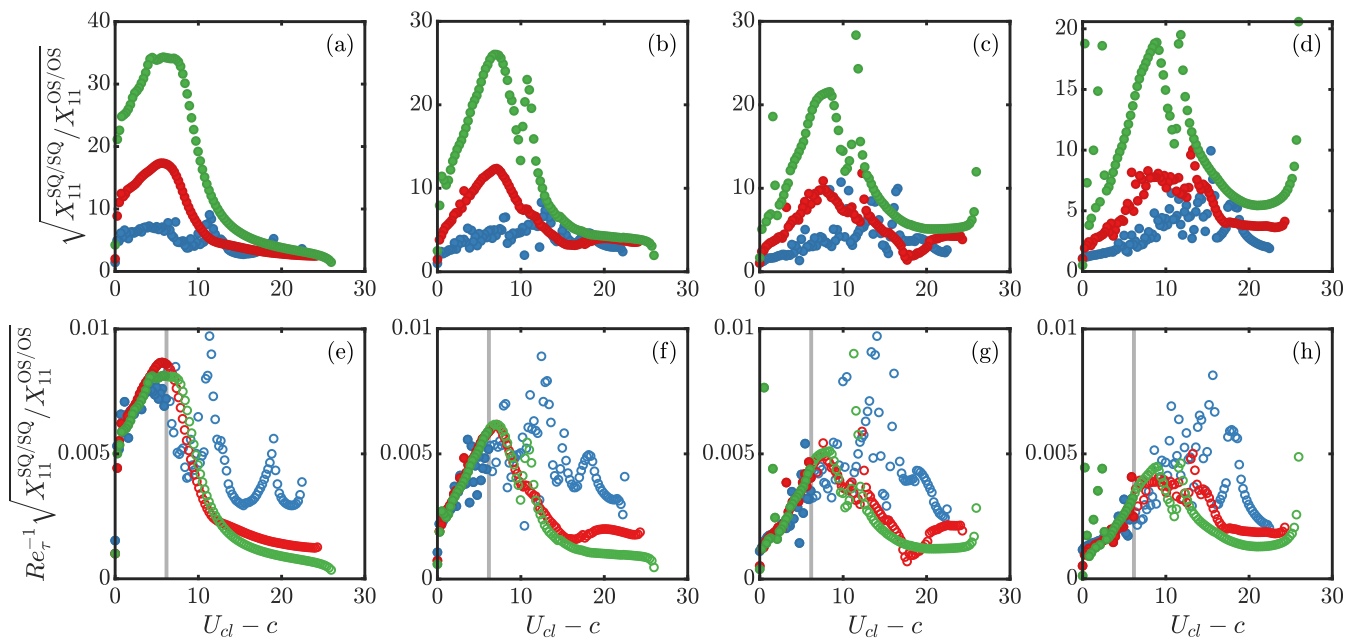

FIG. 12. (a)-(d) Leading weights ratio for $\operatorname{Re}_{\tau}=934$ (blue), $\operatorname{Re}_{\tau}=2003$ (red), and $\operatorname{Re}_{\tau}=4219$ (green) with $N=2$. (e-h) Weights ratio scaled according to Eq. (43). Each column represents a different scaled streamwise wave number $\tilde{k}_{x}$ : (a), (e) $\tilde{k}_{x}=4219$; (b), (f) $\tilde{k}_{x}=8438$; (c), (g) $\tilde{k}_{x}=12657$; (d), (h) $\tilde{k}_{x}=16876$. In all cases the spanwise wave number is $k_{z}=\gamma \tilde{k}_{x} / \operatorname{Re}_{\tau, \min }$, with $\gamma=\sqrt{10}$. Filled circles denote modes belonging to the universal outer class, and the largest outer class wave-speed defect, $U_{\mathrm{CL}}-c=6.17$, is indicated by the gray line.

aspect ratio $\gamma=\sqrt{10}$. In agreement with Eq. (43), the data from all three Reynolds numbers show reasonable collapse onto a single curve for $U_{\mathrm{CL}}-c \lesssim 6.17$ when scaled by $\mathrm{Re}_{\tau}^{-1}$, as seen in the bottom row of Fig. 12.

\section{Geometrically self-similar class}

The self-similar resolvent modes in the logarithmic region of the mean velocity profile belong to hierarchies parametrized by the critical layer location $y_{c}$ [25]. The corresponding length scales along a hierarchy are

$$
\check{k}_{x}=y_{c}^{+} y_{c} k_{x}, \quad \check{k}_{z}=y_{c} k_{z}, \quad \check{y}=y / y_{c},
$$

and the self-similar class wave parameters are

$$
\mathscr{S}_{h}:\left\{\begin{array}{l}
16.4 \lesssim c \lesssim U_{c l}-6.17 \\
c=U\left(y_{c}^{+}\right)=\kappa^{-1} \log y_{c}^{+}+B, \\
k_{z} / k_{x} \gtrsim \gamma
\end{array}\right.
$$

where $\kappa$ is the Kármán constant. Note that the lower wave-speed bound for the self-similar class, $c=$ 16.4 , is the same as the upper limit for the inner class modes, i.e., the beginning of the logarithmic region is taken to be $y^{+}=100$. As discussed above, recent evidence suggests that this lower limit is $\operatorname{Re}_{\tau}$ dependent [38,39]. However, Moarref et al. [25] demonstrated successful scaling of the selfsimilar modes using the fixed lower limit in Eq. (45), so we continue to use it here. For reference, the beginning of the logarithmic region according to the balance of terms in the mean momentum equation, $y^{+} \approx 2.6 \operatorname{Re}_{\tau}^{1 / 2}$ [38], is indicated by the gray dashed line in Fig. 13. The self-similar modes must also satisfy an aspect ratio constraint. Since the aspect ratio increases like $y_{c}^{+}$along a given hierarchy, it is sufficient that the lowest member on the hierarchy with critical layer $y_{c, l}$ and wave numbers $k_{x, l}$ and $k_{z, l}$ satisfies $k_{z, l} / k_{x, l} \gtrsim \gamma$, where a conservative lower bound is $\gamma \approx \sqrt{10}$ [25]. 

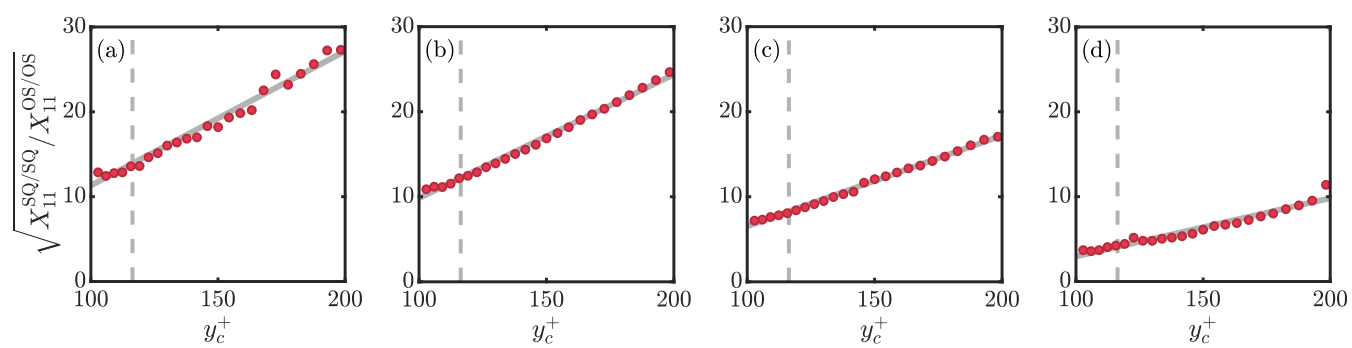

FIG. 13. Leading weights ratio along hierarchies for $\operatorname{Re}_{\tau}=2003$ with $N=2$. Each panel is a different hierarchy, represented by the streamwise wave number at the bottom of the hierarchy: (a) $k_{x, l}=1$; (b) $k_{x, l}=5$; (c) $k_{x, l}=10$; (d) $k_{x, l}=20$. In all cases the spanwise wave number at the bottom of the hierarchy is $k_{z, l}=\gamma k_{x, l}$, with $\gamma=5$. The solid gray lines are the least-squares linear fits, with slopes (a) 0.158 , (b) 0.145 , (c) 0.107 , and (d) 0.069 . The dashed gray lines are $y^{+}=2.6 \mathrm{Re}_{\tau}^{1 / 2} \approx 116$.

Using Eq. (44) and continuity, as well as the orthonormality constraint on the resolvent modes, it follows that

$$
\hat{\boldsymbol{u}}=y_{c}^{-1 / 2}\left(\begin{array}{c}
\check{u} \\
y_{c}^{+-1} \check{v} \\
y_{c}^{+-1} \check{w}
\end{array}\right),
$$

where $(\breve{.})$ indicates a quantity that is approximately $y_{c}$ and $\operatorname{Re}_{\tau}$ invariant for modes belonging to the self-similar class. Collapse of the response modes for this scaling class is shown in Appendix B.

The $y_{c}$-scaled versions of the weighted resolvent operators are

$$
\begin{gathered}
\left(\begin{array}{c}
\mathcal{F}_{v} \mathcal{H}_{v v} \mathcal{F}_{v}^{-1} \\
\mathcal{F}_{\eta} \mathcal{H}_{\eta v} \mathcal{F}_{v}^{-1}
\end{array}\right)=\left(\begin{array}{c}
y_{c} y_{c}^{+} \check{\mathcal{F}}_{v} \check{\mathcal{H}}_{v v} \check{\mathcal{F}}_{v}^{-1} \\
y_{c} y_{c}^{+2} \check{\mathcal{F}}_{\eta} \check{\mathcal{H}}_{\eta v} \check{\mathcal{F}}_{v}^{-1}
\end{array}\right), \\
\mathcal{F}_{\eta} \mathcal{H}_{\eta \eta} \mathcal{F}_{\eta}^{-1}=y_{c} y_{c}^{+} \check{\mathcal{F}}_{\eta} \check{\mathcal{H}}_{\eta \eta} \check{\mathcal{F}}_{\eta}^{-1},
\end{gathered}
$$

so that their leading singular values scale as

$$
\sigma_{j}^{\mathrm{OS}}=y_{c} y_{c}^{+2} \check{\sigma}_{j}^{\mathrm{OS}}, \quad \sigma_{j}^{\mathrm{SQ}}=y_{c} y_{c}^{+} \check{\sigma}_{j}^{\mathrm{SQ}} .
$$

As with the outer class modes, the scaling of the OS singular values is only expected to hold for the first several modes.

Analysis of the scaled energy density matrices (see Appendix B) gives the streamwise energy spectrum as

$$
\begin{aligned}
E_{u u}= & y_{c} y_{c}^{+4} \operatorname{Re}\left\{\operatorname{tr}\left(\check{\boldsymbol{A}}_{u u}^{\mathrm{OS} / \mathrm{OS}} \boldsymbol{X}^{\mathrm{OS} / \mathrm{OS}}\right)\right\}+2 y_{c} y_{c}^{+3} \operatorname{Re}\left\{\operatorname{tr}\left(\check{\boldsymbol{A}}_{u u}^{\mathrm{SQ} / \mathrm{OS}} \boldsymbol{X}^{\mathrm{OS} / \mathrm{SQ}}\right)\right\} \\
& +y_{c} y_{c}^{+2} \operatorname{Re}\left\{\operatorname{tr}\left(\check{\boldsymbol{A}}_{u u}^{\mathrm{SQ} / \mathrm{SQ}} \boldsymbol{X}^{\mathrm{SQ} / \mathrm{SQ}}\right)\right\},
\end{aligned}
$$

where the $y_{c}$ dependence of the right-hand side is explicit, except for the unscaled weights matrices $\boldsymbol{X}^{\mathrm{X} / \mathrm{Y}}$. Balancing all three terms requires

$$
\left|\frac{\chi_{j}^{\mathrm{SQ}}}{\chi_{j}^{\mathrm{OS}}}\right| \sim y_{c}^{+} .
$$

The ratio $\sqrt{\boldsymbol{X}_{11}^{\mathrm{SQ} / \mathrm{SQ}} / \boldsymbol{X}_{11}^{\mathrm{OS} / \mathrm{OS}}}$ is plotted along several hierarchies with different $k_{x, l}$ and $k_{z, l}$ for $\operatorname{Re}_{\tau}=2003$ in Fig. 13. In this case, to have a sufficient number of wave speeds belonging to $\mathscr{S}_{h}$ while keeping the size of the optimization problem manageable, the matching of the DNS spectra is only enforced for $y_{\min }^{+}=100 \leqslant y^{+} \leqslant 0.1 \operatorname{Re}_{\tau}=y_{\max }^{+}$, with $N_{c}=25$. The scaling given by 
Eq. (50) is clearly demonstrated, as the data in all cases exhibit a linear dependence on $y_{c}^{+}$to within a good approximation. In all cases shown the aspect ratio at the bottom of the hierarchies is $\gamma=5$. Similar results are obtained using different aspect ratios, provided that $\gamma \gtrsim \sqrt{10}$ [25]. The slopes of the lines are observed to decrease with increasing $k_{x, l}$. Although not shown here, the slopes tend to increase with increasing $\gamma$.

\section{DISCUSSION AND CONCLUSIONS}

A low-order representation of the time-averaged energy spectra of turbulent channel flow based on the resolvent analysis framework was presented. The resolvent mode weights, which encode information about the nonlinear interactions in the flow, were determined empirically by computing the weights that minimize the deviation between the resolvent spectra and spectra obtained from DNS using a convex optimization scheme. The present approach is a modification of previous work [18], with the major difference being the incorporation of a recently proposed alternative decomposition of the resolvent operator into two distinct families of modes, referred to as the Orr-Sommerfeld and Squire families [22].

It was demonstrated that the alternative OS-SQ decomposition results in a dramatic improvement in the performance of the representation. This improvement is attributed to the isolation of the $v$ response in the OS family, which enables the $\eta$ response of the SQ family to compete with the large $\eta$ response generated by the OS modes. Furthermore, for certain values of the wave parameters, the leading modes of the standard resolvent operator are almost identical to the leading modes of the OS resolvent, so that the mechanisms encoded in the SQ operator are essentially neglected; this helps explain the relatively poor performance of the representation obtained using the standard resolvent. A decomposition of the statistics into contributions from the OS modes, SQ modes, and an interaction between the two families supports this claim and is in agreement with results from DNS at $\operatorname{Re}_{\tau}=185$ [23]. It was further shown that the competition between the OS and SQ modes can be interpreted as a phase difference, and that this phase difference is speculated to be close to $\pi$ over large portions of spectral space.

Next, the scaling of the leading singular values for the OS and SQ families were derived for the inner, outer, and geometrically self-similar universal classes of resolvent modes [25]. For the inner class, both sets of singular values scale as $\mathrm{Re}_{\tau}^{-1}$. For the outer and self-similar classes, the OS singular values are larger than the SQ ones by a factor of $\mathrm{Re}_{\tau}$ and $y_{c}^{+}$, respectively. Interestingly, this large difference in amplification suggests that modes in these classes are likely to be among those for which the OS and standard resolvent modes are nearly identical. Indeed, the scaling of the leading OS singular values is the same as that for the leading singular values of the standard resolvent for the outer and self-similar classes. For the inner class, the scaling for both the OS and SQ singular values matches the standard resolvent. Combining the scalings with the hypothesis that the competition between SQ and OS modes discussed above remains relevant for arbitrary $\operatorname{Re}_{\tau}$ and throughout, the flow domain was used to derive the relative scalings of the OS and SQ weights in each of the universal classes. The scaling predictions were tested against the optimized weights, and, with the exception of high aspect ratio modes of the inner class localized very near the wall, good agreement with the computed optimal weights was found for each of the universal classes.

The results presented herein have several important implications for equation-driven modeling of turbulent channel flow. The first is that partitioning the resolvent operator into Orr-Sommerfeld and Squire subsystems, originally presented in the context of ECS [22], is also advantageous in terms of its ability to develop compact representations of fully turbulent channel flow at high Reynolds number. Furthermore, it provides valuable insight into the complex dynamics by identifying the competition mechanism between the OS and SQ modes, which has ramifications for modeling nonlinear interactions. Specifically, considering that for large $\operatorname{Re}_{\tau}$ the OS singular values in the logarithmic and outer regions of the flow are much larger than the SQ ones, it may be tempting from a modeling perspective to neglect the SQ family of modes. However, doing so does not take into account the relative scaling of the forcing terms $\hat{g}_{v}$ and $\hat{g}_{\eta}$ in Eq. (12)—it implicitly assumes they 


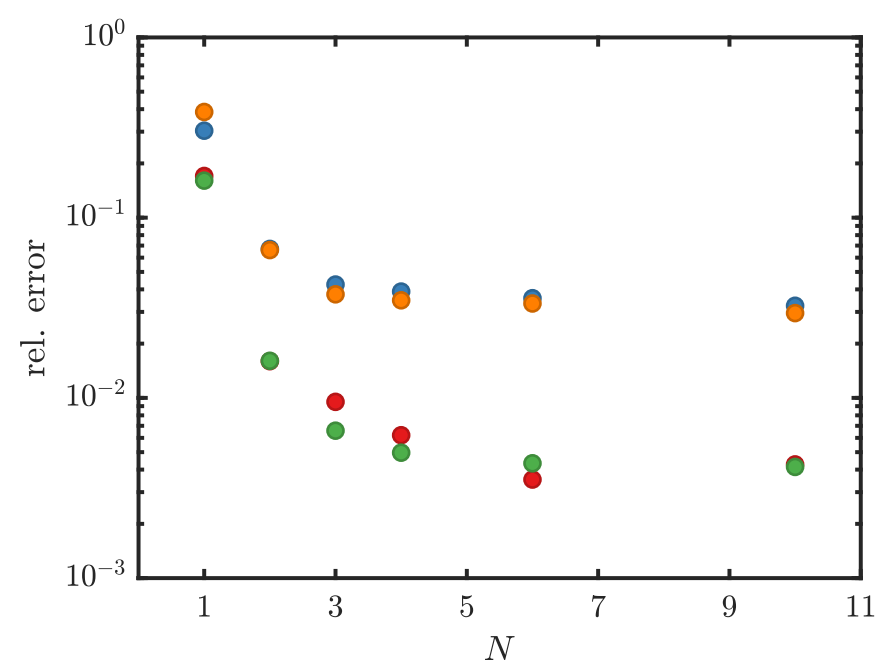

FIG. 14. Relative errors in $\left\langle u^{2}\right\rangle$ (blue), $\left\langle v^{2}\right\rangle$ (red), $\left\langle w^{2}\right\rangle$ (green), and $\langle-u v\rangle$ (orange), as a function of $N^{\mathrm{OS}}=$ $N^{\mathrm{SQ}}=N$.

remain of the same order. The present results indicate that this is not the case. In fact, the scaling results of the weights for all of the classes can be summarized as $\left|\chi_{j}^{\mathrm{SQ}} / \chi_{j}^{\mathrm{OS}}\right| \sim \sigma_{j}^{\mathrm{OS}} / \sigma_{j}^{\mathrm{SQ}}$.

Though the absolute scalings of the weights were not determined, the present paper can be considered a starting point to guide further modeling efforts toward quantifying nonlinear interactions in turbulent channel flow. For instance, it is particularly intriguing that, as discussed in Sec. III A, the $v$ statistics depend only on the OS modes. Consequently, if the scaling of the OS weights can be determined from these, empirically or otherwise, then the results given in Sec. IV can be used to determine the scaling of the SQ weights, effectively reducing the number of unknowns by half. Then a single computation at a relatively low Reynolds number could be combined with the scalings to make predictions of the spectra at Reynolds numbers that are currently unattainable by DNS.

Taken together, the results point to the competition between the OS and SQ modes being an important mechanism in turbulent channel flow that should be respected in order to accurately model the statistics. We hypothesize that if this mechanism could be interrupted the dynamics, and consequently the statistics, of the system would be significantly different. This line of inquiry is the subject of ongoing work.

\section{ACKNOWLEDGMENTS}

The support of AFOSR under Grant No. FA 9550-16-1-0361 and ONR under Grant No. N0001417-1-3022 is gratefully acknowledged. Additionally, the authors would like to thank Javier Jiménez for making the spectra for the $\operatorname{Re}_{\tau}=934$ and 2003 simulations publicly available, as well as Adrián Lozano-Durán for sharing the spectra for $\operatorname{Re}_{\tau}=4219$.

\section{APPENDIX A: BEHAVIOR AT LARGE $\lambda_{x}$}

As discussed in Sec. III A, the most significant discrepancies between the DNS and OS-SQ representation of the 1D spectra shown in Fig. 1 occur in $k_{x} E_{u u}$ at large $\lambda_{x}^{+}$and $y^{+} \lesssim 100$ and $-k_{x} E_{u v}$ at large $\lambda_{x}^{+}$and $y^{+} \lesssim 50$. Furthermore, these errors do not improve considerably with an increasing number of modes, as demonstrated in Fig. 14, which shows a slow decrease in the error for $N^{\mathrm{OS}}=$ $N^{\mathrm{SQ}}=N>4$. The reason for the persisting error is that for large $\lambda_{x}^{+}$there is significant energetic content below the peaks of the lowest wave-speed modes, which typically sit around $y^{+} \approx 40-50$ 


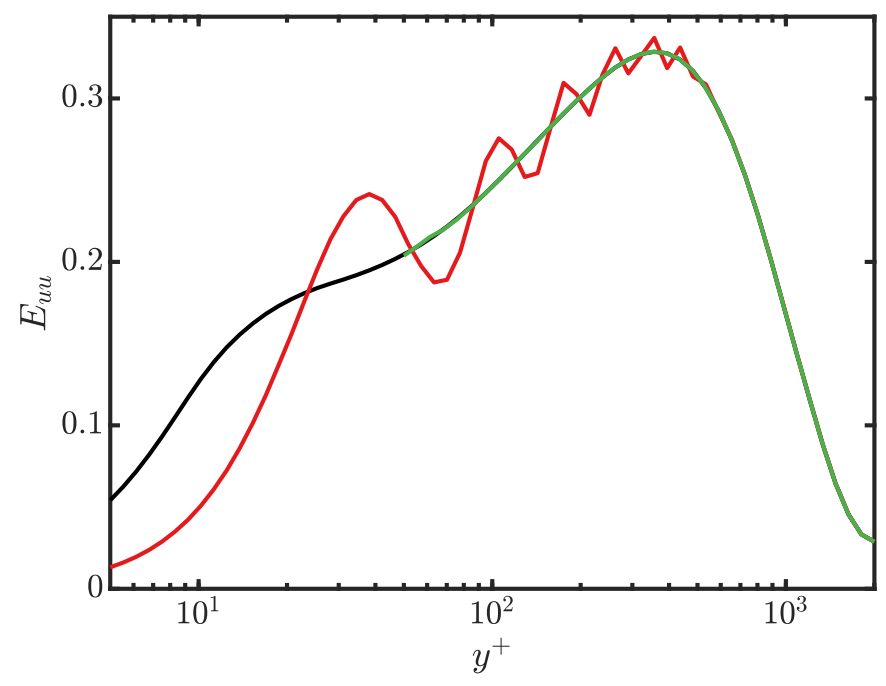

FIG. 15. Comparison of DNS (black) and the optimization results with $y_{\min }^{+}=5$ (red) and $y_{\min }^{+}=50$ (green) using $N^{\mathrm{OS}}=N^{\mathrm{SQ}}=6$ modes for $\left(\lambda_{x}^{+}, \lambda_{z}^{+}\right) \approx\left(3.83 \times 10^{4}, 2.78 \times 10^{3}\right)$.

for $\mathrm{Re}_{\tau}=2003$ [42]. Thus, trying to match near the wall results in overcompensation at larger $y^{+}$. This is illustrated for the representative wavelengths $\left(\lambda_{x}^{+}, \lambda_{z}^{+}\right) \approx\left(3.83 \times 10^{4}, 2.78 \times 10^{3}\right)$ in Fig. 15. To confirm that this is indeed the cause, Fig. 15 also shows the result of the optimization with $y_{\min }^{+}=50$, in which case the large oscillations disappear.

The near-wall errors eventually diminish as the number of modes tends to infinity. However, the fact that the spectra for these wave numbers are not well represented by a low-rank approximation suggests that the response modes may not be the most efficient basis. Indeed, Rosenberg et al. [43] outline conditions under which the flowfields around a cylinder and for channel ECS are more compactly represented by the response to the forcing generated by the leading response at a different wave-number triplet; it is possible that the present case is a similar situation, but the number of triadic interactions that would have to be accounted for in fully turbulent flow significantly complicates matters. It has also recently been shown that augmentation of Eq. (4) with an eddy viscosity improves the representation of large-scale structures [8,9,44]. Both approaches attempt to constrain the forcing, the former by using triadic interactions to identify which scales are most important, and the latter by choosing to only directly model the large-scale coherent motions. However, as pointed out in Sec. I, such nonlinear interactions are incompatible with the turbulent mean velocity profile when an eddy viscosity is included.

\section{APPENDIX B: RESOLVENT SCALING IN THE UNIVERSAL CLASSES}

\section{Response and forcing mode collapse}

Figure 16 shows examples of the collapse of response and forcing modes under inner, outer, and geometrically self-similar scalings.

For a particular $k_{x}^{+}, k_{z}^{+}$, and $c \in \mathscr{S}_{i}$, the magnitudes of the leading vorticity response modes of the OS and SQ resolvent operators and their corresponding forcing modes for the three $\mathrm{Re}_{\tau}$ in Fig. 10 are shown in Figs. 16(a) and 16(b), respectively, using the scalings derived in Eqs. (31)-(33). For comparison, the leading response and forcing mode for the standard resolvent operator are also shown; as discussed in Sec. III A, $\psi_{\eta}$ and $\phi_{v}$ are indistinguishable from the OS modes.

Figures 16(c) and 16(d) show the equivalent mode shapes for a particular $\left(k_{x}, k_{z}, c\right) \in \mathscr{S}_{o}$, using the scalings derived in Eqs. (39)-(41). Again, $\psi_{\eta}$ and $\phi_{v}$ are indistinguishable from the OS modes. 

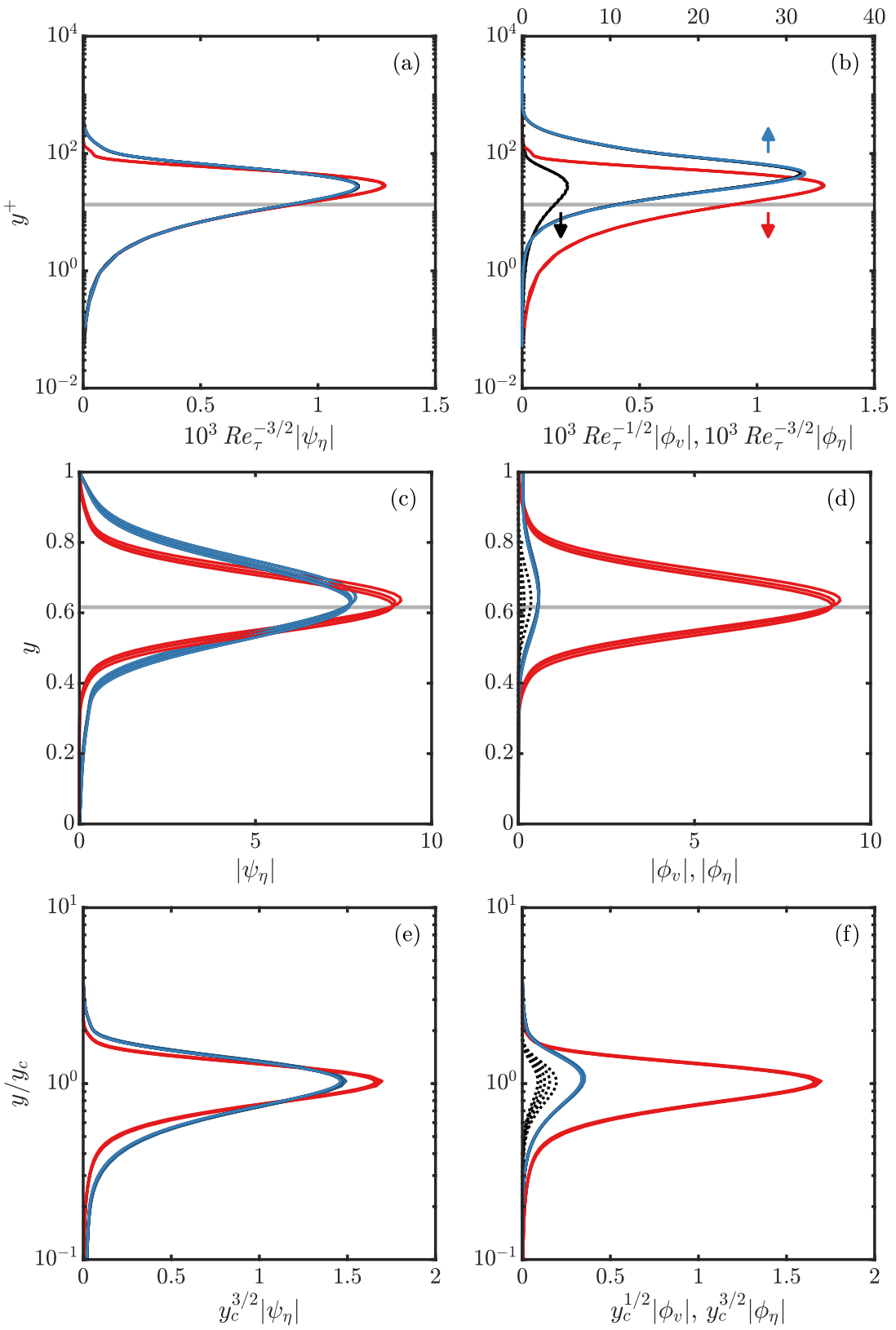

FIG. 16. Magnitudes of the scaled OS (blue), SQ (red), and standard (black) resolvent modes. The gray line indicates the location of the critical layer, where appropriate. Left column: Scaled leading vorticity response modes. Right column: Scaled leading forcing mode magnitudes. (a), (b) Inner scaling class modes for the three $\operatorname{Re}_{\tau}$ shown in Fig. 10, with $k_{x}^{+}=1 / 934, k_{z}^{+}=10 k_{x}^{+}$, and $c=10$, with the relevant axes indicated by the arrows in (b). (c), (d) Outer scaling class for the three $\operatorname{Re}_{\tau}$ shown in Fig. 10, with $\tilde{k}_{x}=934, \tilde{k}_{z}=\gamma=$ $1.5 \sqrt{10}$, and $U_{\mathrm{CL}}-c=1$. (e), (f) Self-similar scaling for five members of a hierarchy at $\operatorname{Re}_{\tau}=2003$, with $k_{x, l}=10$ and $k_{z, l}=10^{3 / 2}$. For the mode combinations shown here, $\psi_{\eta}$ and $\phi_{v}$ in each scaling regime for the standard resolvent are indistinguishable from the OS modes. $\phi_{\eta}$ for the standard resolvent are shown in black dotted lines. 
Apparent from these figures is that the scaling of the outer class modes is only approximate. Recalling that the derivation of such universal classes relies on universal behavior of the mean profile, this is not surprising, since it is clear from Fig. 10(b) that the mean profiles for the three $\operatorname{Re}_{\tau}$ do not collapse perfectly for $y>0.1$. Additionally, $\phi_{\eta}$ for the standard resolvent does not obey the same scaling as $\phi_{\eta}^{\mathrm{SQ}}$. Indeed, using the scaling of the standard resolvent in primitive variables presented in Sharma et al. [40], it can be shown that $\phi_{\eta}=O\left(\mathrm{Re}_{\tau}^{-1}\right)$.

The magnitudes of the leading vorticity response modes of the OS and SQ resolvent operators and their corresponding forcing modes for five members of a particular hierarchy at $\operatorname{Re}_{\tau}=2003$ are shown in Figs. 16(e) and 16(f), respectively, using the scalings derived in Eqs. (46)-(48). Again, $\psi_{\eta}$ and $\phi_{v}$ from the standard resolvent operator are indistinguishable from the OS modes. Here again, $\phi_{\eta}$ for the standard resolvent and $\phi_{\eta}^{\mathrm{SQ}}$ exhibit different scalings, with the standard resolvent $\phi_{\eta}=O\left(y_{c}^{-3 / 2} y_{c}^{+-1}\right)$.

\section{Energy density-matrix scalings}

Expressions for the inner-scaled energy density matrices were given by substituting Eqs. (31) and (33) into Eq. (17). We give here the equivalent expressions for the outer and self-similar scaling classes.

Substituting Eqs. (39) and (41) into Eq. (17), the outer-scaled energy density matrices are

$$
\begin{array}{ll}
\boldsymbol{A}_{u u, i j}^{\mathrm{OS} / \mathrm{OS}}=\operatorname{Re}_{\tau}^{4} \tilde{\boldsymbol{A}}_{u u, i j}^{\mathrm{OS} / \mathrm{OS}}, \quad \boldsymbol{A}_{u u, i j}^{\mathrm{OS} / \mathrm{SQ}}=\operatorname{Re}_{\tau}^{3} \tilde{\boldsymbol{A}}_{u u, i j}^{\mathrm{OS} / \mathrm{SQ}}, \quad \boldsymbol{A}_{u u, i j}^{\mathrm{SQ} / \mathrm{SQ}}=\operatorname{Re}_{\tau}^{2} \tilde{\boldsymbol{A}}_{u u, i j}^{\mathrm{SQ} / \mathrm{SQ}}, \\
\boldsymbol{A}_{v v, i j}^{\mathrm{OS} / \mathrm{OS}}=\operatorname{Re}_{\tau}^{2} \tilde{\boldsymbol{A}}_{v v, i j}^{\mathrm{OS} / \mathrm{OS}}, \\
\boldsymbol{A}_{w w, i j}^{\mathrm{OS} / \mathrm{OS}}=\operatorname{Re}_{\tau}^{2} \tilde{\boldsymbol{A}}_{w w, i j}^{\mathrm{OS} / \mathrm{OS}}, \quad \boldsymbol{A}_{w w, i j}^{\mathrm{OS} / \mathrm{SQ}}=\operatorname{Re}_{\tau} \tilde{\boldsymbol{A}}_{w w, i j}^{\mathrm{OS} / \mathrm{SQ}}, \quad \boldsymbol{A}_{w w, i j}^{\mathrm{SQ} / \mathrm{SQ}}=\tilde{\boldsymbol{A}}_{w w, i j}^{\mathrm{SQ} / \mathrm{SQ}}, \\
\boldsymbol{A}_{u v, i j}^{\mathrm{OS} / \mathrm{OS}}=\operatorname{Re}_{\tau}^{3} \tilde{\boldsymbol{A}}_{u v, i j}^{\mathrm{OS} / \mathrm{OS}}, \quad \boldsymbol{A}_{u v, i j}^{\mathrm{OS} / \mathrm{SQ}}=\operatorname{Re}_{\tau}^{2} \tilde{\boldsymbol{A}}_{u v, i j}^{\mathrm{OS} / \mathrm{SQ}} .
\end{array}
$$

Substituting Eqs. (46) and (48) into Eq. (17), we obtain the scaled energy density matrices:

$$
\begin{array}{ll}
\boldsymbol{A}_{u u, i j}^{\mathrm{OS} / \mathrm{OS}}=y_{c} y_{c}^{+4} \check{\boldsymbol{A}}_{w u, i j}^{\mathrm{OS} / \mathrm{OS}}, \quad \boldsymbol{A}_{u u, i j}^{\mathrm{OS} / \mathrm{SQ}}=y_{c} y_{c}^{+3} \check{\boldsymbol{A}}_{w u, i j}^{\mathrm{OS} / \mathrm{SQ}}, \quad \boldsymbol{A}_{u u, i j}^{\mathrm{SQ} / \mathrm{SQ}}=y_{c} y_{c}^{+2} \check{\boldsymbol{A}}_{u u, i j}^{\mathrm{SQ} / \mathrm{SQ}}, \\
\boldsymbol{A}_{v v, i j}^{\mathrm{OS} / \mathrm{OS}}=y_{c} y_{c}^{+2} \check{\boldsymbol{A}}_{v v, i j}^{\mathrm{OS} / \mathrm{OS}}, \\
\boldsymbol{A}_{w w, i j}^{\mathrm{OS} / \mathrm{OS}}=y_{c} y_{c}^{+2} \check{\boldsymbol{A}}_{w w, i j}^{\mathrm{OS} / \mathrm{OS}}, \quad \boldsymbol{A}_{w w, i j}^{\mathrm{OS} / \mathrm{SQ}}=y_{c} y_{c}^{+} \check{\boldsymbol{A}}_{w w, i j}^{\mathrm{OS} / \mathrm{SQ}}, \quad \boldsymbol{A}_{w w, i j}^{\mathrm{SQ} / \mathrm{SQ}}=y_{c} \check{\boldsymbol{A}}_{w w, i j}^{\mathrm{SQ} / \mathrm{SQ}}, \\
\boldsymbol{A}_{u v, i j}^{\mathrm{OS} / \mathrm{OS}}=y_{c} y_{c}^{+3} \check{\boldsymbol{A}}_{u v, i j}^{\mathrm{OS} / \mathrm{OS}}, \quad \boldsymbol{A}_{u v, i j}^{\mathrm{OS} / \mathrm{SQ}}=y_{c} y_{c}^{+2} \check{\boldsymbol{A}}_{u v, i j}^{\mathrm{OS} / \mathrm{SQ}} .
\end{array}
$$

[1] L. N. Trefethen, A. E. Trefethen, S. Reddy, and T. A. Driscoll, Hydrodynamic stability without eigenvalues, Science 261, 578 (1993).

[2] M. R. Jovanović and B. Bamieh, Componentwise energy amplification in channel flows, J. Fluid Mech. 534, 145 (2005).

[3] P. J. Schmid, Nonmodal stability theory, Annu. Rev. Fluid Mech. 39, 129 (2007).

[4] J. C. Del Alamo and J. Jiménez, Linear energy amplification in turbulent channels, J. Fluid Mech. 559, 205 (2006).

[5] C. Cossu, G. Pujals, and S. Depardon, Optimal transient growth and very large-scale structures in turbulent boundary layers, J. Fluid Mech. 619, 79 (2009).

[6] A. P. Willis, Y. Hwang, and C. Cossu, Optimally amplified large-scale streaks and drag reduction in turbulent pipe flow, Phys. Rev. E 82, 036321 (2010).

[7] Y. Hwang and C. Cossu, Linear non-normal energy amplification of harmonic and stochastic forcing in the turbulent channel flow, J. Fluid Mech. 664, 51 (2010). 
[8] S. J. Illingworth, J. P. Monty, and I. Marusic, Estimating large-scale structures in wall turbulence using linear models, J. Fluid Mech. 842, 146 (2018).

[9] A. Madhusudanan, S. J. Illingworth, and I. Marusic, Coherent large-scale structures from the linearized Navier-Stokes equations, J. Fluid Mech. 873, 89 (2019).

[10] A. Towne, A. Lozano-Durán, and X. Yang, Resolvent-based estimation of space-time flow statistics, J. Fluid Mech. 883, A17 (2020).

[11] S. B. Vadarevu, S. Symon, S. J. Illingworth, and I. Marusic, Coherent structures in the linearized impulse response of turbulent channel flow, J. Fluid Mech. 863, 1190 (2019).

[12] A. Zare, M. R. Jovanović, and T. T. Georgiou, Colour of turbulence, J. Fluid Mech. 812, 636 (2017).

[13] B. J. McKeon and A. S. Sharma, A critical-layer framework for turbulent pipe flow, J. Fluid Mech. 658, 336 (2010).

[14] M. T. Landahl, A wave-guide model for turbulent shear flow, J. Fluid Mech. 29, 441 (1967).

[15] B. J. McKeon, A. S. Sharma, and I. Jacobi, Experimental manipulation of wall turbulence: A systems approach, Phys. Fluids 25, 031301 (2013).

[16] F. Gómez, H. M. Blackburn, M. Rudman, A. S. Sharma, and B. J. McKeon, A reduced-order model of three-dimensional unsteady flow in a cavity based on the resolvent operator, J. Fluid Mech. 798, R2 (2016).

[17] S. Beneddine, D. Sipp, A. Arnault, J. Dandois, and L. Lesshafft, Conditions for validity of mean flow stability analysis, J. Fluid Mech. 798, 485 (2016).

[18] R. Moarref, M. R. Jovanović, J. A. Tropp, A. S. Sharma, and B. J. McKeon, A low-order decomposition of turbulent channel flow via resolvent analysis and convex optimization, Phys. Fluids 26, 051701 (2014).

[19] S. Hoyas and J. Jiménez, Scaling of the velocity fluctuations in turbulent channels up to $\operatorname{Re}_{\tau}=2003$, Phys. Fluids 18, 011702 (2006).

[20] A. Towne, O. T. Schmidt, and T. Colonius, Spectral proper orthogonal decomposition and its relationship to dynamic mode decomposition and resolvent analysis, J. Fluid Mech. 847, 821 (2018).

[21] A. S. Sharma, R. Moarref, B. J. McKeon, J. S. Park, M. D. Graham, and A. P. Willis, Low-dimensional representations of exact coherent states of the Navier-Stokes equations from the resolvent model of wall turbulence, Phys. Rev. E 93, 021102(R) (2016).

[22] K. Rosenberg and B. J. McKeon, Efficient representation of exact coherent states of the Navier-Stokes equations using resolvent analysis, Fluid Dyn. Res. 51, 011401 (2019).

[23] K. Rosenberg, Resolvent-based modeling of flows in a channel, Ph.D. thesis, California Institute of Technology, 2018.

[24] P. J. Schmid and D. S. Henningson, Stability and Transition in Shear Flows (Springer, New York, 2001).

[25] R. Moarref, A. S. Sharma, J. A. Tropp, and B. J. McKeon, Model-based scaling of the streamwise energy density in high-Reynolds-number turbulent channels, J. Fluid Mech. 734, 275 (2013).

[26] A. S. Sharma and B. J. McKeon, On coherent structure in wall turbulence, J. Fluid Mech. 728, 196 (2013).

[27] V. Juttijudata, J. L. Lumley, and D. Rempfer, Proper orthogonal decomposition in Squire's coordinate system for dynamical models of channel turbulence, J. Fluid Mech. 534, 195 (2005).

[28] J. LeHew, M. Guala, and B. McKeon, A study of the three-dimensional spectral energy distribution in a zero pressure gradient turbulent boundary layer, Exp. Fluids 51, 997 (2011).

[29] Y. Huang and D. P. Palomar, Rank-constrained separable semidefinite programming with applications to optimal beamforming, IEEE Trans. Signal Process. 58, 664 (2009).

[30] J. A. Weideman and S. C. Reddy, A MATLAB differentiation matrix suite, ACM Trans. Math. Softw. 26, 465 (2000).

[31] N. Halko, P.-G. Martinsson, and J. A. Tropp, Finding structure with randomness: Probabilistic algorithms for constructing approximate matrix decompositions, SIAM Rev. 53, 217 (2011).

[32] A. Lozano-Durán and J. Jiménez, Effect of the computational domain on direct simulations of turbulent channels up to $\operatorname{Re}_{\tau}=4200$, Phys. Fluids 26, 011702 (2014).

[33] J. F. Gibson, Channelflow: A spectral Navier-Stokes simulator in $\mathrm{C}++$, Technical report, University of New Hampshire, 2014, Channelflow. org. 
[34] S. S. Toedtli, M. Luhar, and B. J. McKeon, Predicting the response of turbulent channel flow to varyingphase opposition control: Resolvent analysis as a tool for flow control design, Phys. Rev. Fluids 4, 073905 (2019).

[35] M. Grant and S. Boyd, CVX: Matlab software for disciplined convex programming, version 2.1, http: //cvxr.com/cvx (2014).

[36] S. T. M. Dawson and B. J. McKeon, On the shape of resolvent modes in wall-bounded turbulence, J. Fluid Mech. 877, 682 (2019).

[37] A. A. Townsend, Equilibrium layers and wall turbulence, J. Fluid Mech. 11, 97 (1961).

[38] J. Klewicki, P. Fife, and T. Wei, On the logarithmic mean profile, J. Fluid Mech. 638, 73 (2009).

[39] I. Marusic, J. P. Monty, M. Hultmark, and A. J. Smits, On the logarithmic region in wall turbulence, J. Fluid Mech. 716, R3 (2013).

[40] A. S. Sharma, R. Moarref, and B. J. McKeon, Scaling and interaction of self-similar modes in models of high Reynolds number wall turbulence, Phil. Trans. R. Soc. A 375, 20160089 (2017).

[41] R. Moarref, A. S. Sharma, J. A. Tropp, and B. J. McKeon, A foundation for analytical developments in the logarithmic region of turbulent channels, arXiv:1409.6047.

[42] R. Moarref, A. S. Sharma, J. A. Tropp, and B. J. McKeon, On effectiveness of a rank-1 model of turbulent channels for representing the velocity spectra, in Proceedings of the 43rdAIAA Fluid Dynamics Conference (AIAA, 2013), https://arc.aiaa.org/doi/pdf/10.2514/6.2013-2480

[43] K. Rosenberg, S. Symon, and B. J. McKeon, Role of parasitic modes in nonlinear closure via the resolvent feedback loop, Phys. Rev. Fluids 4, 052601 (2019).

[44] Y. Hwang, Mesolayer of attached eddies in turbulent channel flow, Phys. Rev. Fluids 1, 064401 (2016). 